University of Nebraska - Lincoln

DigitalCommons@University of Nebraska - Lincoln

Faculty Publications, Department of Physics and Astronomy

Research Papers in Physics and Astronomy

2019

Duality and free energy analyticity bounds for few-body Ising models with extensive homology rank

\author{
Yi Jiang \\ University of California, Riverside \\ Ilya Dumer \\ University of California, Riverside, dumer@ee.ucr.edu \\ Alexey Kovalev \\ University of Nebraska - Lincoln, alexey.kovalev@unl.edu \\ Leonid Pryadko \\ University of California, Riverside, leonid.pryadko@ucr.edu
}

Follow this and additional works at: https://digitalcommons.unl.edu/physicsfacpub

Part of the Quantum Physics Commons

Jiang, Yi; Dumer, llya; Kovalev, Alexey; and Pryadko, Leonid, "Duality and free energy analyticity bounds for few-body Ising models with extensive homology rank" (2019). Faculty Publications, Department of Physics and Astronomy. 305.

https://digitalcommons.unl.edu/physicsfacpub/305

This Article is brought to you for free and open access by the Research Papers in Physics and Astronomy at DigitalCommons@University of Nebraska - Lincoln. It has been accepted for inclusion in Faculty Publications, Department of Physics and Astronomy by an authorized administrator of DigitalCommons@University of Nebraska - Lincoln. 


\section{Duality and free energy analyticity bounds for few-body Ising models with extensive homology rank}

Cite as: J. Math. Phys. 60, 083302 (2019); https://doi.org/10.1063/1.5039735

Submitted: 10 May 2018 . Accepted: 10 July 2019 . Published Online: 02 August 2019

Yi Jiang, llya Dumer, Alexey A. Kovalev (D), and Leonid P. Pryadko (D)
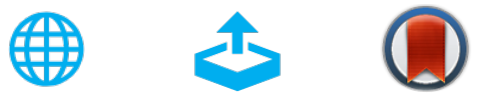

View Online

Export Citation

\section{ARTICLES YOU MAY BE INTERESTED IN}

Invariant measures for the box-ball system based on stationary Markov chains and periodic Gibbs measures

Journal of Mathematical Physics 60, 083301 (2019); https://doi.org/10.1063/1.5095622

Quasi-locality bounds for quantum lattice systems. I. Lieb-Robinson bounds, quasi-local maps, and spectral flow automorphisms

Journal of Mathematical Physics 60, 061101 (2019); https://doi.org/10.1063/1.5095769

Are almost-symmetries almost linear?

Journal of Mathematical Physics 60, 082101 (2019); https://doi.org/10.1063/1.5087539

Journal of

Mathematical Physics

READY TO SUBMIT YOUR RESEARCH?

There are many reasons to publish with us.

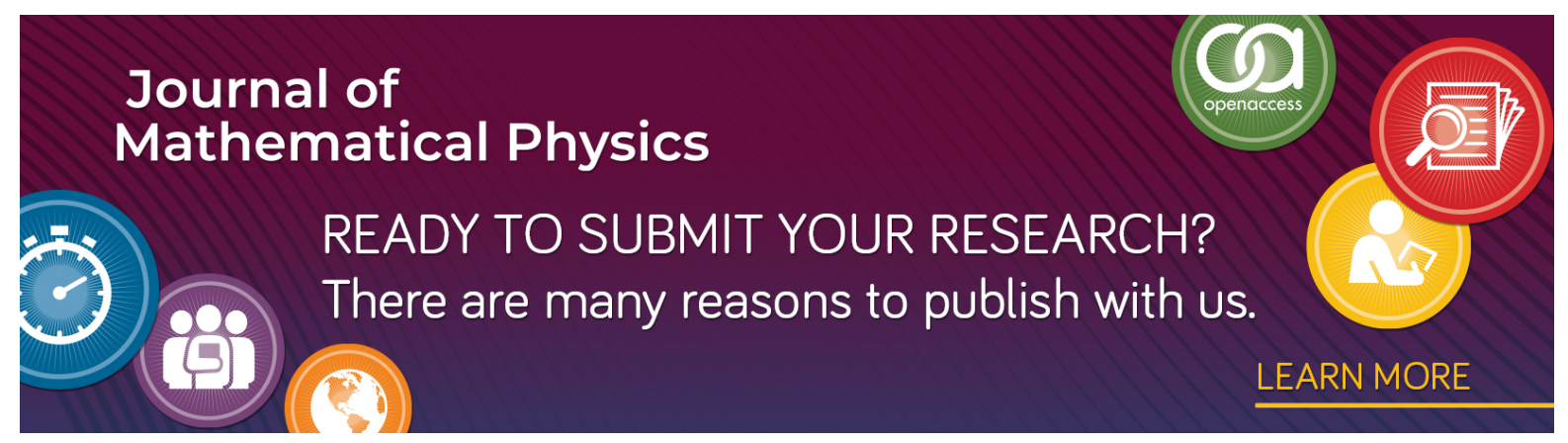




\title{
Duality and free energy analyticity bounds for few-body Ising models with extensive homology rank
}

\author{
Cite as: J. Math. Phys. 60, 083302 (2019); doi: 10.1063/1.5039735 \\ Submitted: 10 May 2018 - Accepted: 10 July 2019 • \\ Published Online: 2 August 2019
}

Yi Jiang, ${ }^{1}$ Ilya Dumer, ${ }^{2}$ Alexey A. Kovalev, ${ }^{3}$ (D) and Leonid P. Pryadko

\begin{abstract}
AFFILIATIONS
${ }^{1}$ Department of Physics and Astronomy, University of California, Riverside, California 92521, USA

${ }^{2}$ Department of Electrical Engineering, University of California, Riverside, California 92521, USA

${ }^{3}$ Department of Physics and Astronomy and Nebraska Center for Materials and Nanoscience, University of Nebraska, Lincoln, Nebraska 68588, USA
\end{abstract}

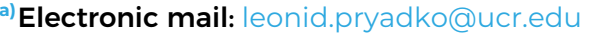

\begin{abstract}
We consider pairs of few-body Ising models where each spin enters a bounded number of interaction terms (bonds) such that each model can be obtained from the dual of the other after freezing $k$ spins on large-degree sites. Such a pair of Ising models can be interpreted as a two-chain complex with $k$ being the rank of the first homology group. Our focus is on the case where $k$ is extensive, that is, scales linearly with the number of bonds $n$. Flipping any of these additional spins introduces a homologically nontrivial defect (generalized domain wall). In the presence of bond disorder, we prove the existence of a low-temperature weak-disorder region where additional summation over the defects has no effect on the free energy density $f(T)$ in the thermodynamical limit and of a high-temperature region where an extensive homological defect does not affect $f(T)$. We also discuss the convergence of the high- and low-temperature series for the free energy density, prove the analyticity of limiting $f(T)$ at high and low temperatures, and construct inequalities for the critical point(s) where analyticity is lost. As an application, we prove multiplicity of the conventionally defined critical points for Ising models on all $\{f, d\}$ tilings of the infinite hyperbolic plane, where $d f /(d+f)>2$. Namely, for these infinite graphs, we show that critical temperatures with free and wired boundary conditions differ, $T_{c}^{(\mathrm{f})}<T_{c}^{(\mathrm{w})}$.
\end{abstract}

Published under license by AIP Publishing. https://doi.org/10.1063/1.5039735

\section{INTRODUCTION}

Singular behavior associated with a phase transition may emerge only in the thermodynamical limit as the system size goes to infinity. One example is spin models on any finite-dimensional lattice, where both the interaction strength and its range are finite. Then, the thermodynamical limit is well defined, thanks to the fact that boundary contribution scales sublinearly with the system size. ${ }^{1}$ Respectively, e.g., in the case of an Ising model, the same transition can be alternatively defined as the temperature where spontaneous magnetization appears, spin susceptibility diverges, spin correlations start to decay exponentially, domain wall tension is lost, or as a singular point of the free energy density. ${ }^{1}$

The situation is different if we have a model on a nonamenable graph characterized by a nonzero Cheeger constant, the lower bound on the perimeter to size ratio for all its finite subgraphs. ${ }^{5,6}$ Examples include infinite transitive expander graphs, such as a degree-regular tree, and regular $\{f, d\}$ tilings of the hyperbolic plane, with $(f-2)(d-2)>2$. Physically, nonamenability of a graph implies that the boundary gives a finite contribution to any bulk average so that both the location of a transition and its properties may depend on both the quantity being probed and the boundary conditions used to define the infinite-graph limit.

A number of general results are known that relate properties of a statistical-mechanical model to amenability/nonamenability of the underlying graph. In particular, for a random walk on a bounded-degree graph, the return probability decays exponentially with time iff the 
graph is nonamenable. In the case of Bernoulli percolation, an infinite cluster is necessarily unique on amenable graphs, but it is conjectured that on any transitive nonamenable graph, there is necessarily an interval where multiple infinite clusters coexist. ${ }^{8-10}$ Among other cases, this conjecture has been verified for planar transitive graphs with bounded-degree duals. ${ }^{11}$ In the case of the Ising model, there is never a phase transition with a finite coupling and a nonzero magnetic field on an amenable transitive graph, while such a transition necessarily exists on any bounded-degree nonamenable graph. ${ }^{12}$ Furthermore, phase transition points in Bernoulli percolation, Ising, and $q$-state Potts models (these have the Fortuin-Kasteleyn random cluster representation with parameters 1, 2, and $q$, respectively ${ }^{13,14}$ ) are known to depend on the boundary conditions when the Cheeger constant is sufficiently large ${ }^{15}$ and on planar nonamenable graphs with regular duals when $q$ is large enough.

From a physics perspective, nonamenable graphs are nonlocal, in the sense that they cannot be embedded in a Euclidean space without infinitely stretching some edges. Most natural geometry for such graphs is hyperbolic, with a constant negative curvature. Interest in quantum field theory models on curved space-time is motivated by quantum gravity and, in particular, the AdS/CFT correspondence. ${ }^{17-23}$ There is an independent interest in models on curved spaces in statistical mechanics and condensed matter communities, e.g., since curvature can serve as an additional parameter to drive the criticality, or as a way to introduce geometrical frustration in toy models of amorphous solids, supercooled liquids, and metallic glasses. ${ }^{24-31}$ Models such as percolation on more general expander graphs and various random graph ensembles are also common in network theory, e.g., such models occurred in relation to internet stability and spread of infectious diseases. ${ }^{32-38}$ Finally, the strongest motivation to study nonlocal Ising models comes from their relation ${ }^{39-41}$ to certain families of finite-rate quantum error-correcting codes (QECCs).

In a companion paper ${ }^{41}$ devoted to error-correcting properties of QECCs, three of us studied pairs of weakly dual few-body Ising models where each spin enters a bounded number of interaction terms (bonds). Each model can be obtained from the exact dual of the other after freezing $k$ spins which enter a large number of bonds. For the related QECC, $k$ is the number of encoded qubits, and its ratio to the number of bonds, $R \equiv k / n$, is the code rate. One can also map such a pair of Ising models to a 2-chain complex $\Sigma$, in which case $k$ is the rank of the first homology group $H_{1}(\Sigma)$. In particular, in Ref. 41, we introduced the homological difference $\Delta F \geq 0$, the difference of the free energies of two models with and without the additional summation over the homological defects, and gave the sufficient conditions for the existence of a low-temperature low-disorder region on the phase diagram where in the large-system limit, $\Delta F=0$.

In the present work, we study duality and phase transitions in general Ising models, focusing on the case where the homology rank $k$ scales linearly with the number of bonds $n$. Our main technical tool is the specific homological difference scaled by the number of bonds, $\Delta f=\Delta F / n$. Upon duality, $\Delta f$ is mapped to $R \ln 2-\Delta f^{*}$, where $\Delta f^{*}$ is the homological difference for the other model in the pair, at the dual temperature. Existence of a low-temperature homological region where asymptotically $\Delta f=0$ implies that at high temperatures, $\Delta f^{*}=R \ln 2$; with $R>0$, this implies the existence of at least two distinct points, where $\Delta f$ is nonanalytic as a function of temperature. Combining with the analysis of convergence of the high-temperature series (HTS) expansion for the free energy density, we obtain several bounds for critical temperatures associated with the nonanalyticity of the limiting free energy densities of the two models. The main result is the inequality for the change of thus defined critical point due to summation over the homological defects.

Second, we discuss applications of these general results to two-body Ising models on transitive graphs, with the infinite graph $G$ obtained as the weak limit of a sequence of finite transitive graphs. Finite rate $R$ implies that the corresponding infinite graph has to be nonamenable. In particular, we prove multiplicity of the conventionally defined critical points for Ising models on all $\{f, d\}$ tilings of the hyperbolic plane with $d f /(d+f)>2$. That is, we show that transition temperatures with wired and free boundary conditions differ, $T_{c}^{(\mathrm{w})}>T_{c}^{(\mathrm{f})}$, which extends the results of Refs. 15, 16, and 42.

The paper is organized as follows: We introduce the notations and review some known facts from the theory of general Ising models and the theory of QECCs in Sec. II. Our results are given in Sec. III, where we first discuss properties of the specific homological difference $\Delta f$, analyze the convergence and analyticity of free energy density for a sequence of weakly dual Ising model pairs, and finally apply the obtained results to Ising models on $\{f, d\}$ tilings of the hyperbolic plane, additionally illustrating the conclusions with numerical simulations. We summarize the results and list some related open questions in Sec. IV. Most of the proofs are given in Appendices A-H.

\section{NOTATIONS AND BACKGROUND}

We consider general Ising models in Wegner's form, ${ }^{43}$ which describes joint probability distribution of $r \equiv|\mathcal{V}|$ Ising spin variables, $S_{v} \in\{-1,1\}$, associated with elements of the vertex set, $\mathcal{V}$,

$$
\operatorname{Prob}_{\mathbf{e}}[\{S\} ; \Theta ; K, h]=\frac{1}{Z} \prod_{b \in \mathcal{B}} e^{K(-1)^{e_{b}} R_{b}} \prod_{v \in \mathcal{V}} e^{h S_{v}},
$$

where each bond $R_{b} \equiv \prod_{v \in \mathcal{V}} S_{v}^{\Theta_{v b}}, b \in \mathcal{B},|\mathcal{B}|=n$, is a product of the spin variables corresponding to nonzero positions in the corresponding column of the $r \times n$ binary coupling matrix $\Theta$, the binary "error" vector e with components $e_{b}, b \in \mathcal{B}$, describes quenched disorder, and the dimensionless coupling coefficients are $K \equiv \beta J$ and $h \equiv \beta h^{\prime}$, where $J$ is the Ising exchange constant, $h^{\prime}$ is the magnetic field, and $\beta \equiv 1 / T$ is the inverse temperature in energy units. The normalization constant in Eq. (1) is the partition function,

$$
Z \equiv Z_{\mathbf{e}}(\Theta ; K, h) \equiv \sum_{\left\{S_{v}= \pm 1\right\}} \prod_{b \in \mathcal{B}} e^{K(-1)^{e_{b}} R_{b}} \prod_{v \in \mathcal{V}} e^{h S_{v}}
$$


The partition function is commonly written in terms of the corresponding logarithm, the dimensionless free energy, $F=-\ln Z$, or the free energy density (per bond), $f=F / n$.

The binary coupling matrix $\Theta$ in Eq. (1) can be interpreted geometrically in terms of a bipartite Tanner graph, ${ }^{44}$ or, equivalently, as the vertex-edge incidence matrix for a hypergraph $\mathcal{H}=(\mathcal{V}, \mathcal{B})$ with vertex set $\mathcal{V}$ and hyperedge (bond) set $\mathcal{B}$, with each hyperedge $b \in \mathcal{B}$ a nonempty subset of the vertex set, $b \subseteq \mathcal{V}$. In comparison, in a (simple undirected) graph $\mathcal{G}=(\mathcal{V}, \mathcal{E})$, each edge $b \in \mathcal{E}$ is an unordered pair of vertices, $b=\{i, j\} \subseteq \mathcal{V}$. The degree $d_{v}$ of a vertex $v \in \mathcal{V}$ in a (hyper)graph is the number of edges that contain $v$; it is equal to the number of nonzero entries in the $v$ th row of the vertex-edge incidence matrix $\Theta$. Similarly, the size of an edge in a hypergraph is called its degree, $d_{b}=|b|, b \in \mathcal{B}$. In a graph, all edges are pairs of vertices, and all columns of the incidence matrix $\Theta$ have exactly two nonzero entries.

The probability distribution (1) can be characterized via the corresponding marginals, the spin correlations

$$
\left\langle S_{\mathcal{A}}\right\rangle \equiv \sum_{\left\{S_{v}= \pm 1\right\}} S_{\mathcal{A}} \operatorname{Prob}_{\mathbf{e}}(\{S\} ; \Theta ; K, h),
$$

where $\mathcal{A} \subseteq \mathcal{V}$ is a set of vertices, $S_{\mathcal{A}}=\prod_{v \in \mathcal{A}} S_{v}$; by convention, $S_{\emptyset}=1$. At $h=0$, on a finite system and with $\mathbf{e}=\mathbf{0}$, nonzero expectation is obtained for the sets (and only the sets) that can be constructed as products of bonds, ${ }^{43}$

$$
S_{\mathcal{A}}=\prod_{b: m_{b} \neq 0} R_{b}=\prod_{v} \prod_{b} S_{v}^{\Theta}{ }_{v b} m_{b},
$$

where bonds in the product correspond to nonzero positions $m_{b} \neq 0$ in the binary vector $\mathbf{m} \in \mathbb{F}_{2}^{n}$ of magnetic charges. A number of correlation inequalities for spin averages have been constructed; see, Refs. 45 and 46. Particularly important for this work are Griffiths-Kelly-Sherman (GKS) inequalities, ${ }^{47,48}$

$$
\begin{aligned}
\left\langle S_{\mathcal{A}}\right\rangle & \geq 0, \\
\left\langle S_{\mathcal{A}} S_{\mathcal{B}}\right\rangle & \geq\left\langle S_{\mathcal{A}}\right\rangle\left\langle S_{\mathcal{B}}\right\rangle,
\end{aligned}
$$

valid in the ferromagnetic case, $\mathbf{e}=\mathbf{0}$, for any $\mathcal{A}, \mathcal{B} \subseteq \mathcal{V}$.

The second GKS inequality (6) can also be written ${ }^{47,48}$ in terms of the derivative of $\left\langle S_{\mathcal{A}}\right\rangle$ with respect to $K_{\mathcal{B}}$, the coupling constant corresponding to the product of spins $S_{\mathcal{B}}$,

$$
\frac{d\left\langle S_{\mathcal{A}}\right\rangle}{d K_{\mathcal{B}}} \geq 0
$$

This implies the monotonicity of any average with respect to all coupling constants and, as a consequence, the existence of two extremal Gibbs states describing (generally different) thermodynamical limit(s) for the Ising model on an infinite hypergraph $\mathcal{H}=(\mathcal{V}, \mathcal{B})$, with free and wired boundary conditions, respectively. Namely, one considers an increasing sequence $\mathcal{V}_{t}, t \in \mathbb{N}$, of sets of vertices, $\mathcal{V}_{t} \mp \mathcal{V}_{t+1} \subset \mathcal{V}$, which converges weakly to $\mathcal{V}=\cup_{t \in \mathbb{N}} \mathcal{V}_{t}$, and the sequence of sub-hypergraphs $\mathcal{H}_{t}=\left(\mathcal{V}_{t}, \mathcal{B}_{t}\right)$ induced by the sets $\mathcal{V}_{t}$. For each $\mathcal{H}_{t}$, consider also the hypergraph $\mathcal{H}_{t}^{\prime}=\left(\mathcal{V}_{t}^{\prime}, \mathcal{B}_{t}^{\prime}\right)$, obtained from $\mathcal{H}$ by contracting all vertices outside $\mathcal{V}_{t}$ into one. Denote the vertex-edge incidence matrices of $\mathcal{H}_{t}$ and $\mathcal{H}_{t}^{\prime}$ as $\Theta_{t}^{\mathrm{f}}$ and $\Theta_{t}^{\mathrm{w}}$, respectively. Here, " $\mathrm{f}$ " and "w" stand for "free" and "wired" boundary conditions in the Ising models (1) defined with the help of these matrices. Clearly, $\mathcal{H}_{t}$ can be obtained from $\mathcal{H}_{t+1}$ by reducing some couplings to zero, while $\mathcal{H}_{t}^{\prime}$ can be obtained from $\mathcal{H}_{t+1}^{\prime}$ by increasing some couplings to infinity. This implies that for any set of vertices $\mathcal{A} \subset \mathcal{V}$, and $t$ large enough so that $\mathcal{A} \subset \mathcal{V}_{t}$, the averages $\left\langle S_{\mathcal{A}}\right\rangle_{t}^{\mathrm{f}} \leq\left\langle S_{\mathcal{A}}\right\rangle_{t}^{\mathrm{w}}$ are, respectively, nondecreasing and nonincreasing with $t$. They are also bounded, which proves the existence of the corresponding pointwise limits, $\left\langle S_{\mathcal{A}}\right\rangle^{\mathrm{f}} \leq\left\langle S_{\mathcal{A}}\right\rangle^{\mathrm{w}}$, at any $K$ and $h$.

The two limits are known to coincide ${ }^{1}$ for degree-limited graphs embeddable in $D$-dimensional space, e.g., the hypercubic lattice $\mathbb{Z}^{D}$. Indeed, the increasing sequence of subgraphs $\mathcal{G}_{t}=\left(\mathcal{V}_{t}, \mathcal{E}_{t}\right)$ can be chosen so that the boundary grows sublinearly with the total number of spins $\left|\mathcal{V}_{t}\right|$. Such a property is violated in the case of a nonamenable graph $\mathcal{G}$, which has a nonzero edge expansion (Cheeger) constant, $\iota_{E}(\mathcal{G})>0$, defined as

$$
\iota_{E}(\mathcal{G}) \equiv \sup _{\mathcal{W} \subset \mathcal{V}:|\mathcal{W}|<\infty} \frac{\left|\partial_{E} \mathcal{W}\right|}{|\mathcal{W}|}
$$

where $\partial_{E}(\mathcal{W})$ is the set of edges connecting $\mathcal{W}$ with its complement, $\mathcal{V} \backslash \mathcal{W}$. The dependence of the critical temperatures (as seen by the magnetization) on the boundary conditions, $T_{c}^{\mathrm{w}}>T_{c}^{\mathrm{f}}$, where the superscripts stand for "wired" and "free" boundary conditions, respectively, is called the "multiplicity" of critical points. ${ }^{15,16,42}$ Examples are the infinite $d$-regular trees $\mathcal{T}_{d}$ [in this case $T_{c}^{\mathrm{f}}=0, T_{c}^{\mathrm{w}}=(d-1)^{-1}$, see, Ref. 5 ], and the regular $\{f, d\}$ tilings $\mathbf{H}(f, d)$ of the infinite hyperbolic plane, $d f /(f+d)>2$, where in each vertex $d$ regular $f$-gons meet. In the latter case, multiplicity of the critical points has been demonstrated for self-dual graphs, $d=f$, and for graphs with a large enough curvature. ${ }^{15,16,42}$ In Sec. III C, we prove the multiplicity of critical points for the Ising model on all hyperbolic tilings $\mathbf{H}(f, d)$ with $d f /(d+f)>2$.

Another important result for the Ising model (1) is the duality transformation. ${ }^{43,49}$ In particular, in the absence of bond disorder, $\mathbf{e}=0$, and at $h=0$, one has 


$$
Z_{\mathbf{0}}(\Theta ; K)=Z_{\mathbf{0}}\left(\Theta^{*} ; K^{*}\right) 2^{r-n_{g}^{*}}(\sinh K \cosh K)^{n / 2},
$$

where $K^{*}$ is the Kramers-Wannier dual of $K$, namely, $\tanh K^{*}=e^{-2 K}$, the degeneracy $n_{g}^{*}=r^{*}-\operatorname{rank} \Theta^{*}\left(2^{n_{g}^{*}}\right.$ is the number of distinct ground-state spin configurations in the dual representation), and $\Theta^{*}$ is a binary $r^{*} \times n$ matrix exactly dual to $\Theta$ (binary rank is used),

$$
\Theta^{*} \Theta^{T}=0, \quad \operatorname{rank} \Theta+\operatorname{rank} \Theta^{*}=n .
$$

Notice that in Eq. (9), and elsewhere in this work, we simplify the notations by suppressing the argument corresponding to a zero magnetic field, $h=0$.

Exact duality also works in the presence of sign bond disorder, except the corresponding bonds ("electric charges") are mapped by duality to extra factors in front of the exponent, "magnetic charges." The resulting expression is not positive-definite and thus cannot be interpreted as a probability measure; instead, it is proportional to the average of a product of the corresponding bonds. The duality in this case reads $^{43}$

$$
\frac{Z_{\mathbf{e}}(\Theta ; K)}{Z_{\mathbf{0}}(\Theta ; K)}=\left\langle\prod_{b \in \mathcal{B}} R_{b}^{e_{b}}\right\rangle_{\Theta^{*} ; K^{*}},
$$

where the average on the right is computed in the dual model with all bonds ferromagnetic, cf. Eq. (4).

There is a natural notion of equivalence between defects $\mathbf{e}$ that produce identical averages in Eq. (11). For the electric charges in the lhs, equivalent are any two defects which differ by a linear combination of rows of $\Theta, \mathbf{e} \simeq \mathbf{e}^{\prime}=\mathbf{e}+\boldsymbol{\alpha} \Theta$, where $\boldsymbol{\alpha}$ is a length- $r$ binary vector. Such defects are related by Nishimori's spin-glass gauge symmetry ${ }^{50}$ generated by local spin flips $\alpha_{v} \in \mathbb{F}_{2}, v \in \mathcal{V}$, and simultaneous update of the components of $\mathbf{e}$ on the adjacent bonds,

$$
S_{v} \rightarrow(-1)^{\alpha_{v}} S_{v}, \quad e_{b} \rightarrow e_{b}^{\prime} \equiv e_{b}+\sum_{v} \alpha_{v} \Theta_{v b} .
$$

For such a defect $\mathbf{e}$, it is convenient to introduce an invariant distance $d_{\mathrm{e}}$, the minimum number of flipped bonds among all defects in the same equivalence class,

$$
d_{\mathrm{e}} \equiv d_{\mathrm{e}}(\Theta)=\min _{\boldsymbol{\alpha}} \operatorname{wgt}(\mathbf{e}+\boldsymbol{\alpha} \Theta),
$$

where $\operatorname{wgt}(\mathbf{e})$ is the Hamming weight. An identical equivalence relation for the magnetic charges which define the product of spins in the rhs of Eq. (11) can be interpreted as a result of introducing a product of (dual) bonds that form a cycle, i.e., does not change the spins that actually enter the average.

For a finite system and a finite $K>0$, both sides of Eq. (11) are strictly positive. The logarithm of the lhs is proportional to the free energy increment due to the addition of the defect,

$$
\delta_{\mathbf{e}} \equiv \delta_{\mathbf{e}}(\Theta ; K) \equiv \ln Z_{\mathbf{0}}(\Theta ; K)-\ln Z_{\mathbf{e}}(\Theta ; K) ;
$$

in turn, it is proportional to dimensionless defect tension,

$$
\tau_{\mathrm{e}} \equiv \tau_{\mathbf{e}}(\Theta ; K) \equiv \delta_{\mathbf{e}}(\Theta ; K) / d_{\mathrm{e}}
$$

Respectively, the scaling of the spin average in the rhs of Eq. (11) with the minimum number of bonds in the product is called the area-law exponent,

$$
\alpha_{\mathrm{e}} \equiv \alpha_{\mathrm{e}}\left(\Theta^{*} ; K^{*}\right)=-d_{\mathrm{e}}^{-1} \ln \left|\prod_{b \in \mathcal{B}} R_{b}^{e_{b}}\right|_{\Theta^{*} ; K^{*}} .
$$

Second, GKS inequality (6) implies subadditivity,

$$
d_{\mathbf{e}_{1}+\mathrm{e}_{2}} \alpha_{\mathrm{e}_{1}+\mathrm{e}_{2}} \leq d_{\mathrm{e}_{1}} \alpha_{\mathrm{e}_{1}}+d_{\mathrm{e}_{2}} \alpha_{\mathrm{e}_{2}} .
$$

Thus, electric-magnetic duality (11) also implies an exact relation between the defect tension and area-law exponent in a pair of dual models,

$$
\tau_{\mathbf{e}}(\Theta ; K)=\alpha_{\mathbf{e}}\left(\Theta^{*} ; K^{*}\right) .
$$

Combined with Eq. (17), this implies subadditivity for defect free energy cost,

$$
\delta_{\mathbf{e}_{1}+\mathbf{e}_{2}} \leq \delta_{\mathbf{e}_{1}}+\delta_{\mathbf{e}_{2}} .
$$

In the special case of a model with two-body couplings defined on a graph $\mathcal{G}=(\mathcal{V}, \mathcal{E})$, a correlation decay exponent can be defined in terms of pair correlations, 


$$
\alpha \equiv \alpha(\mathcal{G} ; K)=\inf _{i, j \in \mathcal{V}}\left[-\frac{\ln \left\langle S_{i} S_{j}\right\rangle}{d_{i j}}\right],
$$

where $d_{i j}$ is the graph distance between $i$ and $j$. Subadditivity (17) implies that the value of $\alpha$ corresponds to that for pairs with large $d_{i j}$ although the decay rate is not necessarily uniform for all pairs of far-separated vertices. In addition, on an infinite graph, we will use

$$
\bar{\alpha} \equiv \bar{\alpha}(\mathcal{G} ; K)=\limsup _{d_{i j} \rightarrow \infty}\left[-\frac{\ln \left\langle S_{i} S_{j}\right\rangle}{d_{i j}}\right] .
$$

The limit here exists since the expression in the square brackets is bounded by $|\ln \tanh K|$. In particular, finite magnetization on a transitive graph, $\left\langle S_{i}\right\rangle=m>0$, implies by the second GKS inequality (6), $\left\langle S_{i} S_{j}\right\rangle \geq m^{2}$; thus, $\bar{\alpha}=0$, which is a stronger statement than just $\alpha=0$.

We are interested in the Ising models (1) with few-body couplings. More specifically, we consider weight-limited Ising models with vertex and bond degrees bounded by some fixed $\ell$ and $m$, respectively, so that $d_{v} \leq m, v \in \mathcal{V}$, and $d_{b} \leq \ell, b \in \mathcal{B}$. With fixed $\ell$ and $m$, we call such a model $(\ell, m)$-sparse. This refers to the sparsity of the corresponding coupling matrix $\Theta: \ell$ and $m$, respectively, are the maximum weights of a column and of a row of $\Theta$.

Furthermore, we would like to consider models whose duals are in the same class of weight-limited Ising models, with some maximum vertex, $\ell^{*}$, and bond, $m^{*}$, degrees. However, such a condition would be very restrictive if one insists on the exact duality (10). For example, in the case of the square-lattice Ising model with periodic boundary conditions on an $L \times L$ square $(\ell=2$ and $m=4)$, the dual model can be chosen to have the same vertex and bond degrees, $\ell^{*}=2$ and $m^{*}=4$, except for $k=2$ additional summations over periodic/antiperiodic boundary conditions in each direction. These summations can be introduced as additional spins entering $d_{v} \geq L$ bonds, where the lower bound is the length of the shortest domain wall on the $L \times L$ square-lattice tiling of a torus. The two additional summations give no contribution to the asymptotic free energy density at $L \rightarrow \infty$, both in the low- and high-temperature phases, and are often ignored.

Such a weak duality with additional defects for models on locally planar graphs can be generalized by considering a pair of weight-limited binary matrices with $n$ columns each, $G$ and $H$, such that their rows be mutually orthogonal, $G H^{T}=0$. Since we do not require exact duality (10), there are exactly

$$
k \equiv n-\operatorname{rank} G-\operatorname{rank} H
$$

distinct defect vectors $\mathbf{c}_{i} \in \mathbb{F}_{2}^{n}, i \in\{1, \ldots, k\}$, which are orthogonal to rows of $H$ and whose nontrivial linear combinations are linearly independent from rows of $G$.

Just as for the spin glasses on locally planar graphs, matrix $H$ can be used to define frustration, $\mathbf{s} \equiv \mathbf{e} H^{T}$, a gauge-invariant characteristic of bond disorder. As common in spin-glass theory, ${ }^{50}$ we will consider independent identically distributed (i.i.d.) components of the quenched disorder vector e such that $e_{b}=1$ with probability $p$. The corresponding averages are denoted with square brackets, $[\cdot]_{p}$.

In theory of quantum error correcting codes, ${ }^{51-54}$ a pair of binary matrices with orthogonal rows, $G H^{T}=0$, can be used to define a Calderbank-Shor-Steane ${ }^{55,56}$ (CSS) stabilizer code $\mathcal{Q}(G, H)$ which encodes $k$ qubits in $n$; see Eq. (22). Such a quantum code has a convenient representation in terms of classical binary codes. Given a matrix $G$ with $n$ columns, one defines the classical code $\mathcal{C}_{G} \subseteq \mathbb{F}_{2}^{n}$, a linear space of dimension rank $G$ generated by the rows of $G$. One also defines the corresponding dual code $\mathcal{C}_{G}^{\perp}$ of all vectors in $\mathbb{F}_{2}^{n}$ orthogonal to rows of $G$; such a code is generated by the corresponding dual matrix (10), $\mathcal{C}_{G}^{\perp} \equiv \mathcal{C}_{G^{*}}$. By orthogonality, we necessarily have $\mathcal{C}_{H} \subseteq \mathcal{C}_{G}^{\perp}$ and $\mathcal{C}_{G} \subseteq \mathcal{C}_{H}^{\perp}$, where equality is achieved when the two matrices are exact dual of each other, in which case, $k=0$. The defect vectors $\mathbf{c}$ are nonzero CSS codewords of $G$ type, $\mathbf{c} \in \mathcal{C}_{H}^{\perp} \backslash \mathcal{C}_{G}$; there are exactly $2^{k}-1$ inequivalent (mutually nondegenerate ${ }^{52}$ ) vectors of this type. Similarly, there are also $2^{k}-1$ inequivalent $H$-type vectors $\mathbf{b}$ in $\mathcal{C}_{G}^{\perp} \backslash \mathcal{C}_{H}$, where equivalence is defined in terms of rows of $H, \mathbf{b}^{\prime} \simeq \mathbf{b}$ iff $\mathbf{b}^{\prime}=\mathbf{b}+\alpha^{T} H$. For any quantum code, important parameters are its rate, $R \equiv k / n$, and the distance, $d \equiv \min \left(d_{G}, d_{H}\right)$,

$$
d_{G} \equiv \min _{\mathbf{c} \in \mathcal{C}_{H}^{\perp} \backslash \mathcal{C}_{G}} \operatorname{wgt}(\mathbf{c}), \quad d_{H} \equiv \min _{\mathbf{b} \in \mathcal{C}_{G}^{\perp} \backslash \mathcal{C}_{H}} \operatorname{wgt}(\mathbf{b})
$$

As yet another interpretation of the algebraic structure in the pair of weakly dual Ising models with vertex-bond incidence matrices $G$ and $H$ of dimensions $r \times n$ and $r^{\prime} \times n$, respectively, consider a two-chain complex $\Sigma \equiv \Sigma(G, H)$,

$$
\Sigma: C_{2} \equiv \mathbb{F}_{2}^{r^{\prime}} \stackrel{\partial_{2}}{\rightarrow} C_{1} \equiv \mathbb{F}_{2}^{n} \stackrel{\partial_{1}}{\rightarrow} C_{0} \equiv \mathbb{F}_{2}^{r}
$$

where the modules $C_{j}, j \in\{0,1,2\}$ are the linear spaces of binary vectors with dimensions $r, n$, and $r^{\prime}$, respectively, and the boundary operators $\partial_{1}$ and $\partial_{2}$ are two linear maps defined by the matrices $G$ and $H^{T}$. The required composition property, $\partial_{1} \circ \partial_{2}=0$, is guaranteed by the orthogonality between the rows of $G$ and $H$. The number of independent defect vectors (22) is exactly the rank of the first homology group $H_{1}(\Sigma)$. 


\section{RESULTS}

\section{A. Properties of specific homological difference}

We first quantify the effect of homological defects on the properties of general Ising models. To this end, given a pair of binary matrices $G$ and $H$ with $n$ columns each and mutually orthogonal rows, $G H^{T}=0$, consider the specific homological difference ${ }^{41}$ (per bond),

$$
\begin{aligned}
\Delta f_{\mathrm{e}} & \equiv \Delta f_{\mathbf{e}}(G, H ; K) \\
& =\frac{1}{n}\left\{\ln Z_{\mathbf{e}}\left(H^{*} ; K\right)-\ln Z_{\mathbf{e}}(G ; K)\right\},
\end{aligned}
$$

where, to fix the normalization, the dual matrix $H^{*}$ [see Eq. (10)] is constructed from $G$ by adding exactly $k$ row vectors, ${ }^{57}$ linearly independent inequivalent codewords $\mathbf{c} \in \mathcal{C}_{H}^{\perp} \backslash \mathcal{C}_{G}$. This quantity satisfies the inequalities ${ }^{41}$

$$
\begin{aligned}
0 \leq \Delta f_{0}(G, H ; K) & \leq \Delta f_{\mathrm{e}}(G, H ; K), \\
\Delta f_{0}(G, H ; K) & \leq R \ln 2,
\end{aligned}
$$

where $R \equiv k / n$ and $k$ is the homology rank given by Eq. (22). The lower and the upper bounds are saturated, respectively, in the limits of zero and infinite temperatures. In addition, in the absence of disorder, the specific homological difference is a nonincreasing function of $K$ (and nondecreasing function of $T=J / K)$,

$$
\frac{d}{d K} \Delta f_{0}(G, H ; K) \leq 0
$$

Our starting point is the following theorem (related to Theorem 2 in Ref. 41), proved in Appendix A:

Theorem 1. Consider a sequence of pairs of weakly dual Ising models defined by pairs of finite binary matrices with mutually orthogonal rows, $G_{t} H_{t}^{T}=0, t \in \mathbb{N}$, where row weights of each $H_{t}$ do not exceed a fixed $m$. In addition, assume that the sequence of the CSS distances $d_{G_{t}}$ is increasing. Then, the sequence $\Delta f_{t} \equiv\left[\Delta f_{\mathbf{e}}\left(G_{t}, H_{t} ; K\right)\right]_{p}, t \in \mathbb{N}$, converges to zero in the region

$$
(m-1)\left[e^{-2 K}(1-p)+e^{2 K} p\right]<1 .
$$

Remarks. 1-1. The bound in Theorem 1 guarantees the existence of a homological region where $\Delta f_{t}$ converges to zero. Generally, such a region may be wider than what is granted by the sufficient condition (28). We will denote $K_{h}(G, H ; p)$ the smallest $K>0$ such that the series $\Delta f_{t}$ converges to zero at any $K^{\prime}>K$. The corresponding temperature, $T_{h}(G, H ; p) \equiv J / K_{h}(G, H ; p)$, is the upper boundary for the homological region at this $p$. Equation (28) implies, in particular, that $K_{h}(G, H ; 0) \geq \ln (m-1) / 2$.

1-2. In the homological region, the sequence of the average free energy densities $\left[f_{\mathbf{e}}\left(G_{t}, K\right)\right]_{p}$ converges iff the sequence $\left[f_{\mathbf{e}}\left(H_{t}^{*}, K\right)\right]_{p}$ converges, and the corresponding limits coincide.

1-3. In analogy with Eq. (15), we introduce the defect tension in the presence of disorder,

$$
\tau_{\mathbf{c}, \mathbf{e}} \equiv \tau_{\mathbf{c}, \mathbf{e}}(G ; K) \equiv \frac{1}{d_{\mathbf{c}}}\left\{F_{\mathbf{e}+\mathbf{c}}(G ; K)-F_{\mathbf{e}}(G ; K)\right\}
$$

where $d_{\mathbf{c}} \geq d_{G}$ is the minimum weight of the codeword equivalent to $\mathbf{c} \in \mathcal{C}_{H}^{\perp} \backslash \mathcal{C}_{G}$. While the tension (29) is not necessarily positive, it satisfies the inequalities

$$
\left|\tau_{\mathfrak{c}, \mathbf{e}}\right| \leq \tau_{\mathfrak{c}, 0} \leq 2 K
$$

We also define the weighted average defect tension,

$$
\bar{\tau}_{p} \equiv \frac{\sum_{\mathbf{c} \neq \mathbf{0}} d_{\mathbf{c}}\left[\tau_{\mathbf{c}, \mathbf{e}}\right]_{p}}{\sum_{\mathbf{c} \neq \mathbf{0}} d_{\mathbf{c}}},
$$

where the average is over disorder and the $2^{k}-1$ nontrivial defect classes. This quantity satisfies the following bound in terms of the average homological difference,

$$
\zeta \bar{\tau}_{p} \geq R \ln 2-\left[\Delta f_{\mathbf{e}}\right]_{p}
$$

where the dimensionless constant $\zeta \leq 1 / 2$; see Eq. (B5) in Appendix B. In the homological phase, this gives $\bar{\tau}_{p} \geq 2 R \ln 2$. (A related bound was previously obtained for the boundary of decodable phase in Ref. 40.) 
In the absence of disorder, $\mathbf{e}=\mathbf{0}$, the specific homological difference is self-dual, ${ }^{41}$ up to an exchange of the matrices $G$ and $H$, and an additive constant,

$$
\Delta f_{0}(G, H ; K)=R \ln 2-\Delta f_{0}\left(H, G ; K^{*}\right) .
$$

Comparing with the general inequalities (26), one sees that a point close to the lower bound is mapped to a point close to the corresponding upper bound. This implies a version of Theorem 1 applicable for high temperatures:

Theorem 2. Consider a sequence of pairs of weakly dual Ising models defined by pairs of finite binary matrices with mutually orthogonal rows, $G_{t} H_{t}^{T}=0, t \in \mathbb{N}$, where row weights of each $G_{t}$ do not exceed a fixed $m$, CSS distances $d_{H_{t}}$ are increasing with $t$, and the sequence of CSS rates $R_{t} \equiv k_{t} / n_{t}$ converges, $\lim _{t} R_{t}=R$. Then, for any $K \geq 0$ such that $(m-1) \tanh K<1$, the sequence $\Delta f_{t} \equiv\left[\Delta f_{\mathbf{e}}\left(G_{t}, H_{t} ; K\right)\right]_{p}, t \in \mathbb{N}$, converges to $R \ln 2$.

Remarks. 2-1. Since duality is used in the proof, we had to switch the conditions on the matrices $G_{t}$ and $H_{t}$. Similarly, the bound for $\tanh K$ is the Kramers-Wannier dual of that in Eq. (28) at $p=0$.

2-2. We will call the temperature region where the sequence $\Delta f_{t}$ in Theorem 2 converges to $R \ln 2$ the dual homological region. Given that the homological region in the absence of disorder extends throughout the interval $K \geq K_{h}(G, H)$, the corresponding interval for the dual homological region is $K \leq K_{h}^{*}(H, G)$, where $K^{*}$ denotes the Kramers-Wannier dual; see Eq. (9). Respectively, $T_{h}^{*}(H, G)$ $\equiv J / K_{h}^{*}(H, G)$ is the low temperature boundary of the dual homological region at $p=0$.

2-3. In the dual homological region, the sequence of the free energy densities $f_{0}\left(H_{t}^{*}, K\right)$ converges iff the sequence $f_{0}\left(G_{t}, K\right)$ converges, and the corresponding limits $f_{H^{*}}(K)$ and $f_{G}(K)$ satisfy

$$
f_{G}(K)=f_{H^{*}}(K)+R \ln 2 .
$$

Notice that when both sets of matrices $H_{t}$ and $G_{t}, t \in \mathbb{N}$, have bounded row weights, the same sequence $\Delta f_{\mathbf{0}}\left(G_{t}, H_{t} ; K\right)$ converges to zero in the homological region, $K \geq K_{h}(G, H)$, and to $R \ln 2$ in the dual homological region, $K \leq K_{h}^{*}(H, G)$. Since the magnitude of the derivative of the free energy density with respect to $K$ (proportional to the energy per bond) is bounded, for any $R>0$, this implies the existence of a minimum gap between the boundaries of the homological and the dual homological regions. We have the inequality

$$
K_{h}(G, H)-K_{h}^{*}(H, G) \geq R \ln 2 .
$$

\section{B. Free energy analyticity and convergence}

The end points $T_{h}(G, H)$ and $T_{h}^{*}(H, G)$ of the two flat regions in the temperature dependence of the homological difference $\Delta f_{0}$ are clearly the points of singularity. What is the relation between these points and the singular points of the limiting free energy density in individual models, which are usually associated with phase transitions?

To establish such a relation, let us analyze the convergence of free energy density and the analyticity of the corresponding limit as a function of parameters. To this end, consider the high-temperature series (HTS) expansion of the free energy density (2),

$$
f_{\mathbf{e}}(\Theta ; K, h) \equiv \sum_{s=1}^{\infty} \kappa_{\mathbf{e}}^{(s)}\left(\Theta ; J, h^{\prime}\right) \frac{\beta^{s}}{s !},
$$

where both parameters are scaled with the inverse temperature, $K \equiv \beta J$ and $h \equiv \beta h^{\prime}$. The coefficient in front of $\beta^{s}$ is proportional to an order-s cumulant of energy; it is a homogeneous polynomial of the variables $h^{\prime}$ and $J$ of degree $s$. A general bound on high-order cumulants from Ref. 58 gives the following:

Statement 3. Consider any model in the form (1), with an $(\ell, m)$-sparse $r \times n$ coupling matrix $\Theta$. The coefficients of the HTS expansion of the free energy density satisfy

$$
\left|\kappa_{\mathbf{e}}^{(s)}\left(\Theta ; J, h^{\prime}\right)\right| \leq 2^{s-1} s^{s-2} C(\Delta+1)^{s-1} A^{s},
$$

where $A \equiv \max \left(|J|,\left|h^{\prime}\right|\right)$ and $(\boldsymbol{a})$ with $J$ and $h^{\prime}$ both nonzero, $\Delta=\ell m$ and $C=r / n+1$, while $(\boldsymbol{b})$ with $h^{\prime}=0, \Delta=(\ell-1) m$ and $C=1$.

Such a bound implies the absolute convergence of the HTS in a finite circle in the complex plane of $\beta$ and, thus, the analyticity of $f_{\mathbf{e}}(\Theta$; $K, h)$ and all of its derivatives as a function of both variables in a finite region with $|K|$ and $|h|$ small enough, in any finite $(\ell, m)$-sparse Ising model, at any given configuration of flipped bonds e. The same is true for the average free energy $\left[f_{\mathbf{e}}\left(\Theta ; J, h^{\prime}\right)\right]_{p}$.

In this region, at $p=0$, convergence and analyticity of the limiting free energy density for models defined by a sequence of binary matrices $\Theta_{t}, t \in \mathbb{N}$, is equivalent to existence of the (pointwise) $\operatorname{limit}_{t} \lim _{t} \kappa_{\mathbf{0}}^{(s)}\left(G_{t} ; J, h^{\prime}\right)$ for the individual coefficients (remember, each of 
them is a homogeneous two-variate polynomial of degree $s$ ). With the help of the cluster theorem for the HTS coefficients, the existence of the limit can be guaranteed by the Benjamini-Schramm convergence ${ }^{59}$ of the corresponding Tanner graphs; see Refs. 60 and 61 for the corresponding discussion for general models with up to two-body couplings. For our present purposes, the following subsequence construction at $h=0$ is sufficient:

Corollary 4. Any infinite sequence of $(\ell, m)$-sparse Ising models, specified in terms of matrices $\Theta_{j}, j \in \mathbb{N}$, has an infinite subsequence $\Theta_{j(t)}$, $t \in \mathbb{N}$, where $j: \mathbb{N} \rightarrow \mathbb{N}$ is strictly increasing, such that (a) for each s, the sequence of the coefficients $\kappa_{0}^{(s)}\left(\Theta_{t} ; J, 0\right)$ converges with $t$, and $(\boldsymbol{b})$ the sequence of free energy densities $f\left(\Theta_{j(t)} ; K\right)$ has a limit, $\varphi_{\Theta}(K)$, which is an analytic function of $K$ in the interior of the circle $|K| \leq\{2 e[(\ell-1) m$ $+1]\}^{-1}$. Here, $e$ is the base of natural logarithm.

Remarks. 4-1. Similar analyticity bounds apply to a very general class of $(\ell, m)$-sparse models with up to $\ell$-body interactions, where each variable is included in up to $m$ interaction terms, and magnitudes of different interaction terms are uniformly bounded: the dependency graph used in the proof can be used in application to all such models. Examples include a variety of discrete models, e.g., Potts and clock models with few-body couplings, as well as compact continuous models with various symmetry groups, Abelian and non-Abelian, where interaction terms are constructed as traces of products of unitary matrices. This is a generalization of the "right" convergence established for models with two-body couplings $(\ell=2)$ in Refs. 60 and 61.

4-2. The subsequence construction is not necessary in the special case where the Tanner graphs defined by the bipartite matrices $\Theta_{t}$ are transitive, with weak infinite-graph limit $\Theta$ and a center $0 \in \mathcal{V}(\Theta)$, such that a ball of radius $\rho_{t}$ in $\Theta_{t}$ is isomorphic to the ball of the same radius centered around 0 in $\Theta$; here, the sequence of the radii is increasing, $\rho_{t+1}>\rho_{t}, t \in \mathbb{N}$. In this case, the cluster theorem ${ }^{62}$ guarantees that the coefficients $\kappa_{s}\left(\Theta_{t}\right)$ do not depend on $t$ for $\rho_{t}>s$.

To make precise statements applicable outside of the convergence radius of the high-temperature series, we need to ensure that a sequence of free energy densities converges. The question of convergence for a general sequence of Ising models being far outside the scope of this work, we will assume the use of yet another subsequence construction to guarantee the existence of the thermodynamical limit for the free energy density. This is based on the following lemma proved in Appendix F.

Lemma 5. Consider a sequence of $r_{t} \times n_{t}$ binary matrices $\Theta_{t}$, where $0<r_{t} \leq n_{t}$, and $t \in \mathbb{N}$. For any $M>0$, define a closed interval $I_{M} \equiv[0, M]$. (a) There exists a subsequence $\Theta_{t(i)}, i \in \mathbb{N}$, where the function $t: \mathbb{N} \rightarrow \mathbb{N}$ is strictly increasing, $t(i+1)>t(i)$ for all $i \in \mathbb{N}$, such that the sequence of Ising free energy densities converges for any $K \in I_{M}, f_{i}(K) \equiv f_{0}\left(\Theta_{t(i)} ; K\right) \rightarrow f(K)$. (b) The limit $f(K)$ is a continuous nonincreasing concave function with left and right derivatives uniformly bounded,

$$
-1 \leq f_{+}^{\prime}(K) \leq f_{-}^{\prime}(K) \leq 0
$$

for all $K \in I_{M}$.

Let us now assume that we have a sequence of pairs of weakly dual weight-limited Ising models which (a) satisfy the conditions of Theorems 1 and 2 with the asymptotic rate $R$, (b) such that the coefficients of the corresponding HTSs converge, so that the sequences of free energy densities $f\left(G_{t} ; K\right)$ and $f\left(H_{t} ; K\right)$ both converge to analytic functions, $\varphi_{G}(K)$ and $\varphi_{H}(K)$, respectively, at $|K|$ sufficiently small (Corollary 4), and, in addition, (c) the sequences of free energy densities both converge on an interval of real axis $I_{M}$, with $M>\ln (m-1) / 2$.

The interval in (c) is such that Theorems 1 and 2 can be used to extend the convergence to the entire real axis; we denote the corresponding limits $f_{G}(K)$ and $f_{H}(K)$. The continuity of the functions $f_{G}(K)$ and $f_{H}(K)$ (and the corresponding duals), along with the inequality (27) which also survives the limit, guarantees that in the range of temperatures between the homological and the dual homological regions, $T_{h}(G, H)<T<T_{h}^{*}(H, G)$, the specific homological difference $\Delta f(K) \equiv f_{G}(K)-f_{H^{*}}(K)$ satisfies the strict inequality

$$
0<\Delta f(K)<R \ln 2 \text {. }
$$

Notice that the existence of the limit on the real axis does not guarantee analyticity which is only guaranteed by condition (b) in a finite vicinity of $K=0$. Hereafter, we will assume that $f_{G}(K)$ is analytic on the interval $0 \leq K<K_{c}(G)$. That is, for any $\epsilon>0$, there exists a simply connected open complex region $\Omega_{\epsilon} \in \mathbb{C}$ which includes the union of the circle of convergence of HTS for $\varphi_{G}(K)$ from Corollary 4 and the interval $I_{M}, M=K_{c}(G)-\epsilon$, such that the sum of HTS series $\varphi_{G}(K)$ can be analytically continued to $\Omega_{\epsilon}$, and the result coincides with the limit $f_{G}(K)$ on the real axis, $K \in I_{M}$. Furthermore, we will assume that $K_{c}(G)$ is the largest value at which this is possible. Such a threshold may arise either (i) because $K_{c}(G)$ is a singular point of $\varphi_{G}(K)$, e.g., the intersection of the natural boundary of $\varphi_{G}(K)$ with the real axis, or (ii) because the limit on the real axis, $f_{G}(K)$, starts to deviate from the result of the analytic continuation. In either case, this guarantees that the limit on the real axis, $f_{G}(K)$, has a singular point of some sort at $K_{c}(G)$.

According to this definition, $T_{c}(G)=J / K_{c}(G)$ is the highest-temperature point of nonanalyticity of the limiting free energy density $f_{G}(K) ; f_{G}(J / T)$ is analytic for $T>T_{c}(G)$. By duality and Theorem $1, f_{G}(K)$ is also analytic at low temperatures. We denote $T_{c}^{\prime}(G) \leq T_{c}(G)$ the lowest-temperature singular point of $f_{G}(J / T)$.

We make similar assumptions about the properties of the limiting free energy density $f_{H}(K)$ and use similar definitions of the critical temperatures $T_{c}^{\prime}(H) \leq T_{c}(H)$ for $f_{H}(J / T)$. We will also use the dual functions, $f_{G^{*}}(K)$ and $f_{H^{*}}(K)$, which coincide with $f_{G}\left(K^{*}\right)$ and 
$f_{H}\left(K^{*}\right)$ up to an addition of analytic functions of $K$; see Eq. (9). The corresponding lowest- and highest-temperature singular points are exchanged by duality, e.g., $T_{c}^{\prime}\left(H^{*}\right)=T_{c}^{*}(H), T_{c}^{\prime}(H)=T_{c}^{*}\left(H^{*}\right)$. Convergence of $\Delta f\left(G_{t}, H_{t} ; K\right)$ to zero implies that $f_{G}(K)=f_{H^{*}}(K)$ for $K>K_{h}(G, H)$; thus, $f_{G}(K)$ is an analytic function in a complex vicinity of any $K>\max \left(K_{c}^{*}(H), K_{h}(G, H)\right)$. Equivalently,

$$
T_{c}^{\prime}(G) \geq \min \left(T_{c}^{*}(H)=T_{c}^{\prime}\left(H^{*}\right), T_{h}(G, H)\right) .
$$

Once we are assured of convergence of the homological difference, the first observation is that the limit, $\Delta f(K)$, is necessarily a strictly convex function at $T_{h}(G, H)$, and a strictly concave function at $T_{h}^{*}(H, G)$, the singular points which are also the boundaries of the region separating the dual homological region at small $K$ and the homological region at large $K$. On the other hand, both $f_{G}(K)$ and $f_{H^{*}}(K)$ are concave functions. Therefore, the convexity at $T_{h}(G, H)$ must originate from $f_{H^{*}}(G, H)$.

Unfortunately, this does not guarantee that $T_{h}(G, H)$ be a singular point of $f_{H^{*}}(K)$. A higher-order phase transition, with a continuous specific heat but discontinuity or divergence in its first or higher derivative, cannot be eliminated on the basis of the general thermodynamical considerations alone. Therefore, we formulate Theorem 6 below (proved in Appendix G) with a list of independently sufficient conditions.

Theorem 6. Let us assume that any one of the following conditions is true:

1. The transition at $T_{c}^{\prime}(G)$ is discontinuous or has a divergent specific heat;

2. The derivative of $\Delta f(K)=f_{G}(K)-f_{H^{*}}(K)$ is discontinuous at $K_{h} \equiv K_{h}(G, H)$, or the derivative of $\Delta f(K)$ is continuous at $K_{h}$, but its second derivative diverges at $K_{h}$;

3. Summation over homological defects does not increase the critical temperature, $T_{c}\left(G^{*}\right) \leq T_{c}(H)$.

Then, the Kramers-Wannier dual of the critical temperatures $T_{c}(H)$ satisfies

$$
T_{c}^{*}(H) \leq T_{h}(G, H)
$$

Remarks. 6-1. We are making the same assumptions about the properties of $f_{H}(K)$, which gives $T_{c}^{*}(G) \leq T_{h}(H, G)$. Combining with Eq. (35), we have

$$
K_{c}\left(H^{*}\right)-K_{c}(G) \geq R \ln 2 .
$$

This implies a strict inequality, $T_{c}(G)>T_{c}\left(H^{*}\right)$, when the homological rank scales extensively, $R>0$, which is superficially similar to the multiplicity of critical points on nonamenable infinite graphs; ${ }^{15,16,42}$ see Sec. II. The difference is that our critical temperatures correspond to points of nonanalyticity of the limiting free energy density in zero magnetic field; we do not have a direct connection to magnetic transitions.

6-2. It is known that stabilizer codes with generators local in $\mathbb{Z}^{D}$ and divergent distances have asymptotically zero rates. ${ }^{63,64}$ This is perfectly consistent with the known fact that weight-limited models local in $\mathbb{Z}^{D}$ have well-defined thermodynamical limits, independent of the boundary conditions. ${ }^{1}$ For example, inequality (42) with $R=0$ is saturated in the case of planar self-dual Ising models, where the transition is in the self-duality point, which is the only nonanalyticity point of the free energy density.

6-3. Most important application of Theorem 6 and Eq. (42) is few-body Ising models that correspond to finite-rate quantum LDPC codes with distances scaling as a power of the code length $n, d \geq A n^{\alpha}$ with $A, \alpha>0$. Examples are quantum hypergraph-product (QHP) and related codes, ${ }^{65-67}$ and higher-dimensional hyperbolic codes. ${ }^{68}$ Because of higher-order couplings, generic mean-field theory gives a discontinuous transition, which is the case of condition 1 in Theorem 6 . The discontinuous nature of the transition has been verified numerically for one class of QHP codes. ${ }^{41}$

6-4. Ising models on expander graphs are known to have mean-field criticality. ${ }^{15,69}$ A combination of an analytic $f_{H^{*}}(K)$ and a finite specific heat jump in $f_{G}(K)$ at $K_{h}(G, H)$ is not eliminated by conditions 1 or 2 . We discuss the important case of Ising models on hyperbolic graphs in Sec. III C.

6-5. GKS inequalities imply that any spin average satisfies $\left\langle S_{\mathcal{A}}\right\rangle_{G ; K} \geq\left\langle S_{\mathcal{A}}\right\rangle_{H^{*} ; K}$. Physically, this ought to be sufficient to guarantee condition 3 , but we are not aware of a general proof.

\section{Application to models on hyperbolic graphs}

\section{Bounds for infinite-graph transition temperatures}

While the inequalities (35) are (42) are certainly important results, they address unconventionally defined critical points. Both the homological critical point, $T_{h}(G, H)$, and the end points of the interval of possible nonanalyticity, $T_{c}^{\prime}(G) \leq T_{c}(G)$, are defined for sequences of Ising models without boundaries. They are not immediately related to the critical temperatures $T_{c}^{\mathrm{f}} \leq T_{c}^{\mathrm{w}}$ defined on related infinite systems in terms of extremal Gibbs states with free/wired boundary conditions.

To bound these critical temperatures, consider a sequence of pairs of weakly dual Ising models which satisfy the conditions of Theorems 1 and 2 with the asymptotic rate $R>0$, with an additional assumption that matrices $G_{t}$ and $H_{t}$ are incidence matrices of graphs, that is, 
they have uniform column weights $\ell=\ell^{*}=2$. In addition, we assume that the graph sequences converge weakly to a pair of infinite transitive graphs, which we denote $\mathcal{G}=(\mathcal{V}, \mathcal{E})$ and $\mathcal{H}=(\mathcal{F}, \mathcal{E})$, where $\mathcal{F}$ is the set of faces in $\mathcal{G}$. Weak convergence is defined as follows: for some chosen vertex $0 \in \mathcal{V}$, there is an increasing sequence $\rho_{t} \in \mathbb{N}$ such that the ball $\mathcal{B}\left(0, \rho_{t}\right) \subset \mathcal{G}$ of radius $\rho_{t}$ centered at 0 , is isomorphic to a ball in $\mathcal{G}_{t}$.

These conditions necessarily imply that matrices $G_{t}$ and $H_{t}$ describe mutually dual locally planar graphs and also that the graphs $\mathcal{G}$ and $\mathcal{H}$ are mutually dual.

Examples of such a sequence are given by sequences of finite hyperbolic graphs constructed ${ }^{27,70}$ as finite quotients of the regular $\{f, d\}$ tilings of the infinite hyperbolic plane, $\mathbf{H}(f, d)$, with $d f /(d+f)>2$. A graph in such a sequence gives a tiling of certain surface, with $d$ regular $f$-gons meeting in each vertex. Hyperbolic graphs have been extensively discussed in relation to quantum error correcting codes. ${ }^{71-77}$ Given such a finite locally planar transitive graph with $n$ edges, the quantum CSS code is a surface code; ${ }^{39,78}$ it is constructed from the vertex-edge and plaquette-edge incidence matrices, $G$ and $H$, respectively. Here, $H$ is also a vertex-edge incidence matrix of a dual graph, which corresponds to the dual tiling $\{d, f\}$ of the same surface. Such a code has the minimal distance scaling logarithmically with $n$, and it encodes $k=2 g=2+n R$ qubits into $n$, where $g$ is the genus of the surface and $R=1-2 / d-2 / f$ is the asymptotic rate.

An extremal Gibbs ensemble on any infinite locally planar transitive graph can be characterized by the average magnetization $m$, the asymptotic correlation decay exponents $\alpha$ [Eq. (20)] and $\bar{\alpha} \geq \alpha$ [Eq. (21)], and similarly defined asymptotic domain wall tensions,

$$
\tau \equiv \tau(\mathcal{G} ; K)=\inf _{\{i, j\} \subset \mathcal{F}} \tau_{\mathbf{e}(i, j)}, \quad \bar{\tau} \equiv \bar{\tau}(\mathcal{G} ; K)=\limsup _{d_{i j} \rightarrow \infty} \tau_{\mathbf{e}(i, j)},
$$

where $\mathbf{e}(i, j)$ is a defect that connects a pair of frustrated plaquettes $i$ and $j$. Generally, $\bar{\alpha}=\alpha=0$, whenever spontaneous magnetization $m$ is nonzero. A nonzero magnetization on a locally planar transitive graph also implies $\tau>0$. (This is a generalization of the result from Ref. 4 , see Appendix H.) Respectively, electromagnetic duality (18) implies

Statement 7. Let $\mathcal{G}$ and $\mathcal{H}$ be a pair of infinite mutually dual locally planar transitive graphs. Denote $T_{c}^{\mathrm{f}}(\mathcal{G})$ and $T_{c}^{\mathrm{w}}(\mathcal{H})$ the critical temperatures of the extremal Gibbs ensembles for Ising models on $\mathcal{G}$ and $\mathcal{H}$ with free and wired boundary conditions, respectively. Then, these temperatures are Kramers-Wannier duals of each other,

$$
T_{c}^{\mathrm{f}}(\mathcal{G})=\left[T_{c}^{\mathrm{w}}(\mathcal{H})\right]^{*}
$$

For each model, in the ordered phase, $T<T_{c}, \bar{\alpha}=0$, and $\tau>0$, while in the disordered phase, $T>T_{c}, \alpha>0$, and $\bar{\tau}=0$.

We can now prove the following:

Theorem 8. For any regular $\{f, d\}$ tiling of an infinite hyperbolic plane, $f d /(f+d)>2$, the critical temperatures of the Ising model with free and wired boundary conditions, $T_{c}^{\mathrm{f}}=1 / K_{c}^{\mathrm{f}}$ and $T_{c}^{\mathrm{w}}=1 / K_{c}^{\mathrm{w}}$, satisfy

$$
K_{c}^{\mathrm{f}}-K_{c}^{\mathrm{w}} \geq R \ln 2, \quad R=1-2 / f-2 / d .
$$

Proof. For any regular $\{f, d\}$ tiling $\mathcal{G} \equiv \mathbf{H}(f, d)$ of the hyperbolic plane, consider a sequence of finite mutually dual locally planar transitive graphs $\mathcal{G}_{t}$ and $\mathcal{H}_{t}$, where the sequence $\mathcal{G}_{t}$ weakly converges to $\mathcal{G}$. The corresponding sequence of incidence matrices satisfies the conditions of Theorems 1 and 2 with the asymptotic rate $R>0$. Transitivity implies that the free energy density converges in a finite circle around $K=0$; see Remark 4-2. While we are not sure of convergence for larger $K$, Lemma 5 guarantees the existence of a subsequence of graphs, and corresponding pairs of incidence matrices $G_{t}, H_{t}, t \in \mathbb{N}$, such that the sequences of free energy densities $f\left(G_{t} ; K\right)$ and $f\left(H_{t} ; K\right)$ converge. For such a sequence, the specific homological difference $\Delta f\left(G_{t}, H_{t} ; K\right)$ also converges, which guarantees $\Delta f<R \ln 2$ outside of the dual homological phase, $K>K_{h}^{*}(H, G)$. Such an inequality implies the existence of an $\epsilon>0$ such that $\Delta f\left(G_{t}, H_{t} ; K\right)<R \ln 2-\epsilon / 2$ at all sufficiently large $t$. In turn, Eq. (32) implies that the average defect tension is bounded away from zero, $\bar{\tau}_{0}\left(G_{t}\right) \geq \epsilon$.

While defects that contribute to the average $\bar{\tau}_{0}\left(G_{t}\right)$ have large weights, we notice that the free energy increment (14) associated with an arbitrary defect is subadditive; see Eq. (19). Thus, a large-weight defect can be separated into smaller pieces; subadditivity (19) ensures that $\max \left(\tau_{\mathbf{e}_{1}}, \tau_{\mathbf{e}_{2}}\right) \geq \tau_{\mathbf{e}_{1}+\mathbf{e}_{2}}$ as long as $d_{\mathbf{e}_{1}+\mathbf{e}_{2}}=d_{\mathbf{e}_{1}}+d_{\mathbf{e}_{2}}$. Thus, if we start with a homological defect with the tension $\tau_{\mathbf{c}} \geq \epsilon>0$, at each division, we can select a piece with the tension not smaller than $\epsilon$. Moreover, since homological defects are cycles on the dual graph, we can first separate $\mathrm{c}$ into simple cycles of weight not smaller than the corresponding CSS distance which increases with $t$ and then cut such a cycle in half to obtain a defect compatible with the definition (43).

Furthermore, GKS inequalities imply that tension $\tau_{\mathrm{e}}$ is monotonously nondecreasing when individual bonds' coupling is increased. Thus, for the same defect $\mathbf{e}=\mathbf{e}(i, j)$ connecting frustrated plaquettes $i$ and $j$ on $\mathcal{G}_{t}$ and on the corresponding planar subgraph with wired boundary conditions, $\tau_{\mathbf{e}(i, j)}\left(G_{t}^{\mathrm{w}} ; K\right) \geq \tau_{\mathbf{e}(i, j)}\left(G_{t} ; K\right) \geq \epsilon$. Subadditivity construction in the previous paragraph guarantees the existence of such pairs for any $t$ and that for any $L>0$ pairs separated by dual-graph distances $d_{i j}>L$ can be chosen with $t$ sufficiently large. Definition (43) then gives $\bar{\tau} \geq \epsilon>0$ for the Ising model with wired boundary conditions on the infinite graph $\mathcal{G}$, at temperatures below the dual homological point, $K>K_{h}^{*}(H, G)$. Necessarily, $K_{c}^{\mathrm{w}}(\mathcal{G}) \leq K_{h}^{*}(H, G)$. 
Duality (44) also ensures that $K_{c}^{\mathrm{f}}(\mathcal{H}) \geq K_{h}(G, H)$; inequality (35) gives Eq. (45).

Remarks. 8-1. An interesting fact about systems with finite rates $R>0$ is that electromagnetic duality (18) does not guarantee that area-law exponent $\alpha_{\mathrm{m}}(G ; K)$ be zero at low temperatures. While "area" is the defect distance $d_{\mathrm{m}}$, the smallest number of bonds in an equivalent defect, the "perimeter" is the number of spins involved in the product, the syndrome weight wgts, where $\mathbf{s}=\mathbf{m} G^{T}$. Standard area/perimeter law argument assumes that perimeter can be parametrically smaller than the area; this is not necessarily true for systems with nonamenable Tanner graphs.

8-2. Even in the case of a pair of locally planar graphs, a linear domain wall e connecting a pair of frustrated plaquettes may have a large perimeter in the dual model because of the additional spins corresponding to the homological defects. Any such defect that crosses the domain wall (changes the sign of the corresponding spin average) increases the perimeter in the dual model. Such additional defects are absent with free boundary conditions as considered in Theorem 8.

\section{Numerical results}

In addition to analytical bounds presented above, we also analyzed numerically Ising models on several finite transitive hyperbolic graphs constructed $^{27,70}$ as finite quotients of the regular $\{5,5\}$ tilings of the infinite hyperbolic plane. We used canonical ensemble simulations with both local Metropolis updates ${ }^{79}$ and Wolff cluster algorithm, ${ }^{80}$ to compute the average magnetization $m=\langle M\rangle / N$, susceptibility $\chi=\left(\left\langle M^{2}\right\rangle-\right.$ $\left.\langle M\rangle^{2}\right) / N T$, average energy per bond $\varepsilon \equiv\langle E\rangle / n$, specific heat $C=\left(\left\langle E^{2}\right\rangle-\langle E\rangle^{2}\right) / n T^{2}$, and the fourth Binder cumulant ${ }^{81} U_{4}=1-\left\langle S^{4}\right\rangle /\left(3\left\langle S^{2}\right\rangle^{2}\right)$. Here, $M=\left|\sum_{i} S_{i}\right|$ is the (magnitude of the) total magnetization, $E=-\sum_{\langle i j\rangle} S_{i} S_{j}$ is the total energy, $N$ and $n$, respectively, denote the number of spins and bonds, and $\langle\cdot\rangle$ denotes the ensemble average. For Metropolis simulations, each run consisted of 128 cooling-heating cycles, with
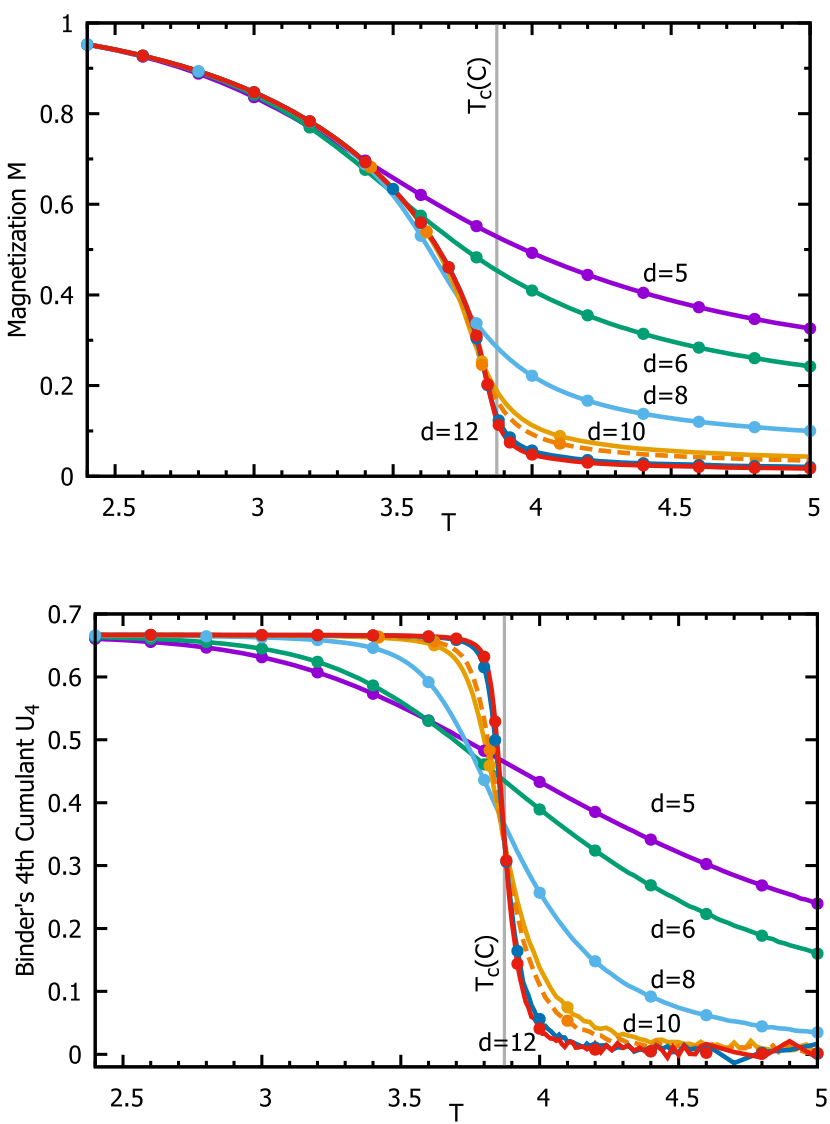

FIG. 1. Average magnetization (top) and Binder's fourth cumulant (bottom) as a function of temperature, for transitive graphs listed in Table I with minimal distances as indicated. Dashed lines show the data for the larger $d=10$ graph. Lines show the data obtained using cluster updates; points show the data from simulations using local Metropolis updates. Vertical line shows the critical temperature $T_{c}(C)$ extrapolated from the positions of the specific heat maxima; see Fig. 5. While both sets of data do cross near $T_{c}(C)$, there is significant drift with increased graph size. In addition, the curves are near parallel which makes reliable extraction of the critical temperature difficult. 


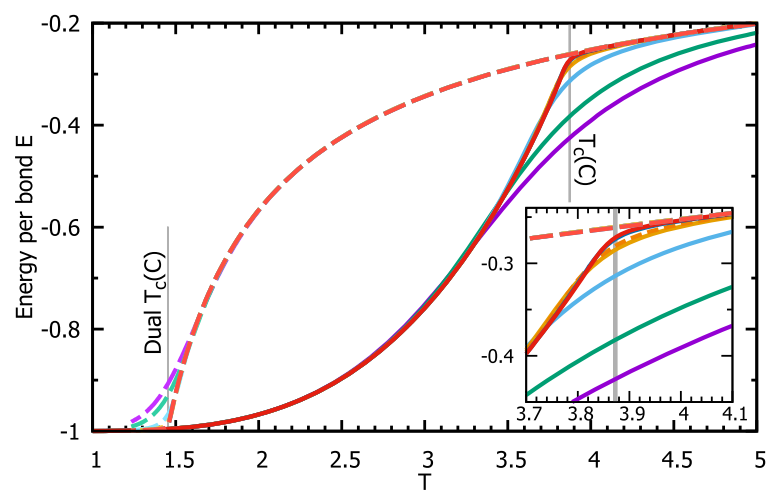

FIG. 2. Solid lines: energy per bond from Wolff cluster calculations as a function of temperature, as in Fig. 1. These data are converted with the help of the exact duality ( 9 ) to give energies in the dual model (long dashes). With increasing graph sizes, the difference between the original and dual energies decreases above the empirically found $T_{c}(C)$ (Fig. 5) and below the corresponding Kramers-Wannier dual, $T_{c}^{*}(C)$. Inset: close up of the plots near $T_{c}(C)$.

1024 full graph sweeps at each temperature, with additional averaging over 64 independent runs of the program. The number of sweeps at each temperature was sufficient to make any hysteresis unnoticeable. For Wolff algorithm simulations, each run consisted of 16 cooling-heating cycles, with 4096 cluster updates at each temperature, and additional averaging over 64 independent runs of the program. The resulting averages are shown in Figs. 1-4, where lines (dots) show the data obtained with cluster (local Metropolis) updates, respectively. The results obtained using the two methods are very close.

The parameters of the graphs used in the simulations are listed in Table I. The first three graphs we obtained from Breuckmann. ${ }^{77}$ We generated the remaining graphs with a custom gap ${ }^{82}$ program, which constructs coset tables of freely presented groups obtained from the infinite van Dyck group $D(5,5,2)=\left\langle a, b \mid a^{5}, b^{5},(a b)^{2}\right\rangle$ [here $a$ and $b$ are group generators, while the remaining arguments are relators which correspond to imposed conditions, $a^{5}=b^{5}=(a b)^{2}=1$ ] by adding one or more relator obtained as a pseudorandom string of generators, until a finite group is obtained. Given such a finite group $\mathcal{D}$, the vertices, edges, and faces are enumerated by the right cosets with respect to the subgroups $\langle a\rangle,\langle a b\rangle$, and $\langle b\rangle$, respectively. The vertex-edge and face-edge incidence matrices $G$ and $H$ are obtained from the coset tables. Namely, nonzero matrix elements are in positions where the corresponding pair of cosets share an element. Finally, the distance $d$ of the CSS code $\mathcal{Q}(G, H)$ was computed using the covering set algorithm, which has the advantage of being extremely fast when distance is small. ${ }^{83,84}$ With the exception of the graph with $n=7440$, the graphs used have the smallest size for the given distance.

The obtained plots of magnetization and Binder's fourth cumulant are shown in Fig. 1; the corresponding curves on largest graphs are nearly indistinguishable, consistent with convergence at large $n$. We note that the crossing point in the Binder's fourth cumulant shows a significant drift with the system size; see the lower plot in Fig. 1 . This is not surprising, given that the original scaling analysis ${ }^{81}$ only applies to locally flat systems, whereas any hyperbolic graph has a uniform negative curvature. On both plots, the curves for larger system

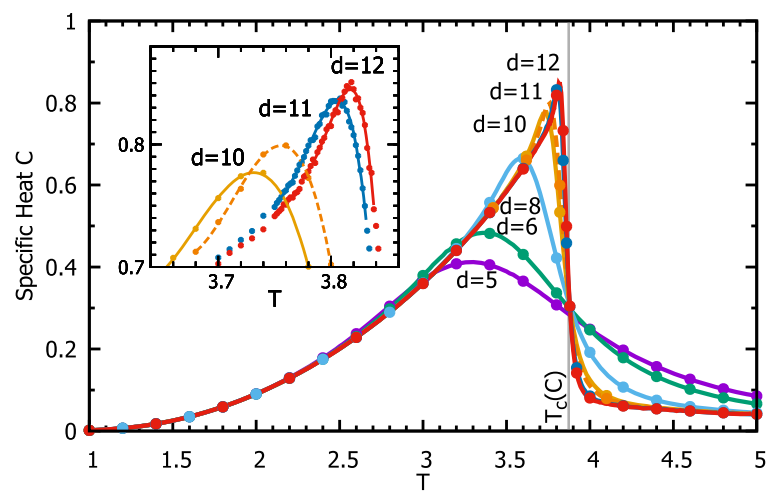

FIG. 3. As in Fig. 1 but for the specific heat. Inset: fitting for maxima. Data points in the inset are from the Wolff cluster calculations, while the lines are obtained using nonlinear fits with general quartic polynomials of the form $y=y_{m}+a_{2}\left(x-x_{m}\right)^{2}+\cdots+a_{4}\left(x-x_{m}\right)^{4}$, which give the coordinates of the maximum $\left(x_{m}, y_{m}\right)$ nearly independent from the rest of the coefficients. 


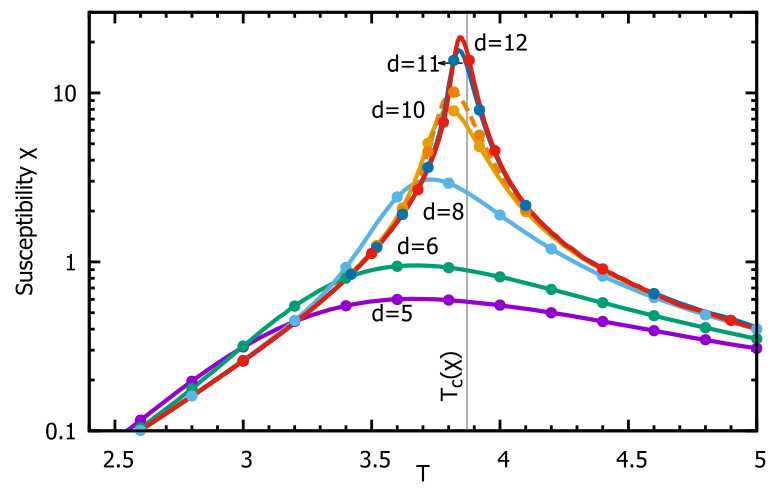

FIG. 4. As in Fig. 1 but for the susceptibility $\chi(T)$, plotted in semilogarithmic scale. The vertical line shows the critical temperature extrapolated from the susceptibility maxima; see Fig. 5.

sizes are near parallel to each other, which makes the identification of the phase transition point from the corresponding crossing points difficult.

Figure 2 shows energy per bond as a function of temperature. To illustrate the properties of the specific homological difference, see Theorems 1 and 2, we also plot the energy per bond of the exact dual models obtained from the same data using $\varepsilon^{*}\left(K^{*}\right)=-\sinh (2 K) \varepsilon(K)$ $-\cosh (2 K)$, derived from Eq. (9). The plot shows that as the size of the graph increases, the difference between the energies $\varepsilon^{*}(T)$ and $\varepsilon(T)$ decreases with increasing graph size both above $T_{c}(C)$ and below the corresponding Kramers-Wannier dual, $T_{c}^{*}(C)$, while a finite difference remains for the intermediate temperatures. This is consistent with the identification $T_{h}^{*}=T_{c}(C)$.

The plots for specific heat $C(T)$ (Fig. 3) and magnetic susceptibility $\chi(T)$ (Fig. 4) show well developed maxima which become sharper and higher with increasing system sizes. Notice that a unique point of divergence of the specific heat necessarily coincides with the dual homological temperature $T_{h}^{*}$.

We obtained the positions of the specific heat and magnetic susceptibility maxima by fitting the data in the vicinity of the corresponding maxima with quartic polynomials, as explained in the caption of Fig. 3. The resulting positions of the maxima are plotted in Fig. 5 as a function of $x=1 / n^{1 / 2}$. The error bars of the positions of the maxima have errors in the third digit; the observed minor scattering of the data is a feature of the corresponding graphs.

While the size dependence is not monotonic in the case of susceptibility maxima, the data points for larger graphs show approximately linear dependence on $x$. Linear extrapolation to infinite size $(x=0)$ gives $T_{c} \approx 3.872 \pm 0.003$ for both sets of data. This value is consistent with the lower bound (42) for the infinite graph with wired boundary conditions, which gives in the present case $T_{c} \geq 2.668$. In comparison, the transition for a square-lattice Ising model is in the self-dual point, $T_{\text {s.d. }}=2 / \ln (1+\sqrt{2}) \approx 2.269$.

We note that even though we expect Ising model on hyperbolic graphs to have mean field criticality, conventional finite size scaling theory does not apply here. In particular, this is seen from the absence of the well defined crossing point in the data for Binder's fourth cumulant; see the lower plot in Fig. 1. Therefore, we had to experiment on how to extrapolate the positions of the maxima to estimate the critical temperature. The scaling with $x=1 / n^{1 / 2}$ was chosen since it gives near identical estimates for the critical temperatures from the maxima of $C(T)$ and $\chi(T)$, cut off at different maximum sizes (we tried $d_{\max }=8$ and above).

We also note that the data show good convergence with increased system size, without the need for the subsequence construction described in Sec. III B.

TABLE I. Parameters of the graphs used in the simulations.

\begin{tabular}{lccc}
\hline \hline Vertices $r$ & Edges $n$ & Homology rank $k$ & CSS distance $d$ \\
\hline 32 & 80 & 18 & 5 \\
60 & 150 & 32 & 6 \\
360 & 900 & 182 & 8 \\
1920 & 4800 & 962 & 10 \\
2976 & 7440 & 1490 & 10 \\
8640 & 21600 & 4322 & 11 \\
12180 & 30450 & 6092 & 12 \\
\hline \hline
\end{tabular}




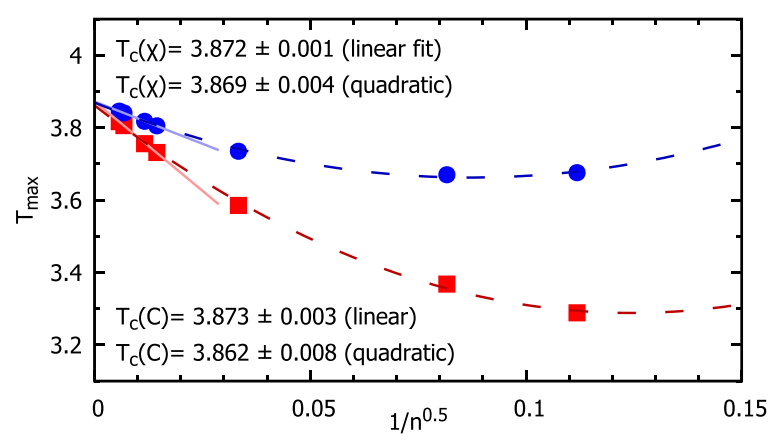

FIG. 5. Extrapolation of the specific heat and susceptibility maxima to infinite system size. Red squares (blue circles) show the positions of the specific heat (susceptibility) maxima extracted from the data in Figs. 3 and 4, respectively, for graphs of different size, plotted as a function of $1 / n^{1 / 2}$, where $n$ is the number of edges in the corresponding graphs; see Table I. Solid (dashed) lines are obtained as linear (quadratic) fits to the data, where only the four leftmost points were used for the linear fits. This results in the extrapolated critical temperature values as indicated.

\section{DISCUSSION}

\section{A. Summary of the results}

We considered pairs of weakly dual Ising models with few-body couplings, defined via sequences of degree-limited bipartite coupling graphs, with the focus on the case where the rank $k$ of the first homology group of the corresponding two-chain complex scales extensively with the system size. This construction is needed to avoid introducing the boundaries, which are known to affect the position of the critical point in nonamenable graphs, and also to connect to applications, e.g., in quantum information theory, where results for large but finite systems are of interest. Here, extensive scaling of $k$ corresponds to quantum error correcting codes with finite rates $R>0$. Important examples include two-body Ising models on families of finite transitive hyperbolic graphs which weakly converge to regular $\{f, d\}$-tilings of the hyperbolic plane with $d f /(d+f)>2$; the corresponding limiting rates $R=1-2 / d-2 / f$ are nonzero.

Our main technical result is Theorem 1, which guarantees the existence of a low-temperature, low-disorder region where homological defects are frozen out-in the thermodynamical limit they have no effect on the free energy density. Duality guarantees the existence of a high-temperature phase where extensive homological defects have near zero free energy cost; see Theorem 2 . At all temperatures below this phase, the average defect tension is nonzero; see Eq. (32).

With the help of duality and a known bound on high-order cumulants, we established the absolute convergence of both the high- and low-temperature series expansions of the free energy density in finite regions which include vicinities of the real temperature axis around the zero and infinite temperatures, respectively. We used a subsequence construction to ensure the convergence of free energy density at all temperatures and defined the critical temperatures as the real-axis points of nonanalyticity of the limiting free energy density. For these critical temperatures, we derived several inequalities, in particular, an analog of multiplicity of the critical points, which guarantees that with $R>0$, critical point of the free energy density is affected by the summation over the topological defects.

As an application of obtained bounds, we proved the multiplicity of phase transitions on all regular tilings $\mathbf{H}(f, d)$ of the infinite hyperbolic plane, $d f /(d+f)>2$.

We also simulated the phase transition on a sequence of self-dual $\{5,5\}$ transitive hyperbolic graphs without boundaries, with up to $n_{\max }=30450$ bonds numerically. Our data show good convergence with increasing system sizes, with a single specific heat maximum which sharpens with the increasing system size. If the corresponding position $T_{c}(C) \approx 3.872 \pm 0.003$ is the only singularity of the free energy, then necessarily it coincides with the dual homological point, $T_{h}^{*}=T_{c}(C)$.

\section{B. Open questions}

1. The rightmost point of the homological region established in Theorem 1 on the $p-T$ plane has the same value $p_{\max }$ as can also be obtained using the energy-based arguments, ${ }^{85}$ which apply at $T=0$. Either of these results also implies ${ }^{40,50}$ that the portion of the Nishimori line at $p<p_{\max }$ is in the homological region. It should be possible to establish the existence of a homological region in the intermediate temperature points, but we could not find the corresponding arguments.

2. The Proof of statement 3 is based on overly generic bounds ${ }^{58}$ for cumulants of a sum of random variables with a given dependency graph. In the case of the Ising model, it should be possible to construct a stronger lower bound for absolute convergence of the HTS. We expect that the same bound as in Theorem 2 should apply. Such a bound would be consistent with that from high-temperature series expansions for spin correlations, ${ }^{86}$ and it would also be consistent with the analysis of the higher-order derivatives of free energy, ${ }^{87}$ as well as the naive expectation that $T_{c}(G)=T_{h}^{*}(H, G)$.

3. In addition to the case in Remark 4-2, the infinite subsequence construction of Corollary 4 is also not needed when the sequence of Tanner graphs has a well defined distributional limit (Benjamini-Schramm or "left" convergence ${ }^{59-61}$ ). Important examples are given 
by the Tanner graphs of hypergraph-product and related codes ${ }^{65,66}$ based on specific families of sparse random matrices. For such sequences, it would be nice to establish the conditions for convergence of the free energy density or spin averages for all $K>0$, to supersede the subsequence construction of Lemma 5.

\section{ACKNOWLEDGMENTS}

L.P.P. is grateful to N. P. Breuckmann for the explanation of the quotient group construction of the hyperbolic graphs. This work was supported in part by the NSF under Grant Nos. PHY-1415600 (A.A.K.), ECCS-1102074 (I.D.), and PHY-1416578 (L.P.P.). L.P.P. also acknowledges hospitality by the Institute for Quantum Information and Matter, an NSF Physics Frontiers Center with support of the Gordon and Betty Moore Foundation.

\section{APPENDIX A: PROOF OF THEOREM 1}

Theorem 1. Consider a sequence of pairs of weakly dual Ising models defined by pairs of finite binary matrices with mutually orthogonal rows, $G_{t} H_{t}^{T}=0, t \in \mathbb{N}$, where row weights of each $H_{t}$ do not exceed a fixed $m$. In addition, assume that the sequence of the CSS distances $d_{G_{t}}$ is increasing. Then, the sequence $\Delta f_{t} \equiv\left[\Delta f_{\mathbf{e}}\left(G_{t}, H_{t} ; K\right)\right]_{p}, t \in \mathbb{N}$, converges to zero in the region

$$
(m-1)\left[e^{-2 K}(1-p)+e^{2 K} p\right]<1 .
$$

The statement of the theorem immediately follows from the following technical lemma, see the proof in Ref. 41:

Lemma 9. Consider a pair of Ising models defined in terms of weight-limited matrices $G$ and $H$ with orthogonal rows such that matrix $H$ has a maximum row weight $m$. Let $d_{G}$ denote the CSS distance (23), the minimum weight of a frustration-free homologically nontrivial defect $\mathbf{c} \in \mathcal{C}_{H}^{\perp} \backslash \mathcal{C}_{G}$. Denote $S \equiv e^{-2 K}(1-p)+e^{2 K} p$, and assume that $(m-1) S<1$. Then, the average homological difference (25) satisfies

$$
[\Delta f(G, H ; K)]_{p} \leq \frac{(m-1)^{d_{G}} S^{d_{G}+1}}{1-(m-1) S} .
$$

\section{APPENDIX B: PROOF OF INEQUALITIES IN SEC. III A}

(i) The proof of the monotonicity of the homological difference (in the absence of flipped bonds),

$$
\frac{d}{d K} \Delta f_{0}(G, H ; K) \leq 0
$$

is similar to the proof ${ }^{88}$ of the monotonicity of the tension. We combine the logarithms in Eq. (25), decompose $Z_{\mathbf{e}}\left(H^{*} ; K\right)$ as a sum of $Z_{\mathbf{c}}(G ; K)$ over nonequivalent codewords $\mathbf{c}$, and write

$$
\frac{d}{d K} \frac{Z_{\mathrm{c}}(G ; K)}{Z_{\mathbf{0}}(G ; K)}=\frac{Z_{\mathrm{c}}(G ; K)}{Z_{\mathbf{0}}(G ; K)} \sum_{b \in \mathcal{B}}\left(\left\langle R_{b}\right\rangle_{\mathrm{c}}-\left\langle R_{b}\right\rangle_{\mathbf{0}}\right) \leq 0 .
$$

The desired inequality (27) follows from the monotonicity of the logarithm.

(ii) The first inequality in

$$
\left|\tau_{\mathbf{c}, \mathbf{e}}\right| \leq \tau_{\mathbf{c}, 0} \leq 2 K
$$

follows from the second GKS inequality ${ }^{47,48}$ applied in the dual system [where, according to electric-magnetic duality, the defect becomes an average of the corresponding product of spins, see Eq. (11)]. Depending on the sign of $\tau_{\mathbf{c}, \mathbf{e}}$, duality gives $\left\langle R_{\mathbf{c}+\mathbf{e}}\right\rangle \geq\left\langle R_{\mathbf{e}}\right\rangle\left\langle R_{\mathbf{c}}\right\rangle$ or $\left\langle R_{\mathbf{e}}\right\rangle \geq\left\langle R_{\mathbf{c}}\right\rangle\left\langle R_{\mathbf{e}+\mathbf{c}}\right\rangle$, where $R_{\mathbf{e}}$ is the product of bonds corresponding to nonzero bits in the binary vector $\mathbf{e}$. The second inequality in Eq. (30), in a more general form, $\tau_{\mathbf{e}} \equiv \tau_{\mathbf{e}, 0} \leq 2 K$, follows from the Gibbs inequality,

$$
F_{\mathbf{e}}(G ; K)-F_{0}(G ; K) \leq 2 K \sum_{b: e_{b} \neq 0}\left\langle R_{b}\right\rangle_{G ; K} \leq 2 K \operatorname{wgt}(\mathbf{e})
$$

if we take a minimal-weight vector equivalent to $\mathbf{e}$, in which case $\operatorname{wgt}(\mathbf{e})=d_{\mathbf{e}}$.

(iii) To prove the lower bound on the average tension,

$$
\zeta \bar{\tau}_{p} \geq R \ln 2-\left[\Delta f_{\mathbf{e}}\right]_{p}
$$


we first define the constant $\zeta$ as the average minimum weight of all $2^{k}$ codewords divided by the code length $n$,

$$
\zeta=\left(2^{k} n\right)^{-1} \sum_{\mathbf{c}} d_{\mathbf{c}}
$$

An upper bound on $\zeta$ can be obtained if we take the codewords $\mathbf{c}$ as linear combinations of $k$ inequivalent codewords $\mathbf{c}_{i}, i \in\{1, \ldots, k\}$ (it is likely that smaller-weight equivalent codewords can be found). In this case, the codewords form a binary code, and the average weight is exactly half of the length $n^{\prime}$ of the code, ${ }^{89}$ where $n^{\prime}=\left|\cup_{i=1}^{k} I\left(\mathbf{c}_{i}\right)\right|$ is the weight of the union of the supports of the basis codewords. Clearly, $n^{\prime} \leq n$, which gives $\zeta \leq 1 / 2$. Combining with a lower bound on the weight of nontrivial codewords, $d_{\mathbf{c}} \geq d_{\mathcal{G}}, \mathbf{c} \neq \mathbf{0}$, we obtain

$$
\frac{1-2^{-k}}{n} d_{G} \leq \zeta \leq \frac{1}{2} .
$$

We now proceed with deriving the inequality (32). Start by expanding $Z_{\mathbf{e}}\left(H^{*} ; K\right)=\sum_{\mathrm{c}} Z_{\mathbf{e}+\mathbf{c}}(G ; K)$, where the summation is over all $2^{k}$ mutually inequivalent codewords $\mathbf{c}$. Each of the terms with $\mathbf{c} \nsucc \mathbf{0}$ can be written in terms of the corresponding tension (29),

$$
Z_{\mathbf{e}+\mathfrak{c}}(G ; K)=e^{-\tau_{\mathfrak{c}, \mathbf{e}}(G ; K) d_{\mathfrak{c}}} Z_{\mathbf{e}}(G ; K) .
$$

Convexity of the exponent gives

$$
\begin{aligned}
\frac{Z_{\mathbf{e}}\left(H^{*} ; K\right)}{Z_{\mathbf{e}}(G ; K)} & =1+\sum_{\mathbf{c} \neq 0} \exp \left(-\tau_{\mathfrak{c}, \mathbf{e}} d_{c}\right) \\
& \geq 2^{k} \exp \left(-2^{-k} \sum_{\mathbf{c}} \tau_{\mathfrak{c}, \mathbf{e}} d_{\mathbf{c}}\right),
\end{aligned}
$$

where for the trivial codeword $\mathbf{c} \simeq \mathbf{0}$, we set $\tau_{0, \mathrm{e}} d_{\mathbf{0}}=0$. Taking the logarithm and rewriting the sum over codewords in terms of the weighted average, with the help of Eq. (B4), we obtain

$$
\Delta F_{\mathbf{e}}(G, H ; K) \geq k \ln 2-\zeta n \frac{\sum_{\mathbf{c} \neq 0} \tau_{\mathbf{c}, \mathbf{e}} d_{\mathbf{c}}}{\sum_{\mathbf{c} \neq 0} d_{\mathbf{c}}} .
$$

Equation (32) trivially follows after averaging over disorder and dividing by $n$.

(iv) The inequality

$$
K_{h}(G, H)-K_{h}^{*}(H, G) \geq R \ln 2
$$

is based on the standard inequality for the derivative of the free energy density, which is just the average energy per bond. For the case of homological difference, we obtain, instead,

$$
\frac{d}{d K} \Delta f(G, H ; K)=\frac{1}{n} \sum_{b \in \mathcal{B}}\left(\left\langle R_{b}\right\rangle_{H^{*} ; K}-\left\langle R_{b}\right\rangle_{G ; K}\right) .
$$

The second term can be obtained from the first by freezing the spins corresponding to homologically nontrivial defects; with the help of GKS inequalities, we obtain

$$
1 \geq\left\langle R_{b}\right\rangle_{G ; K} \geq\left\langle R_{b}\right\rangle_{H^{*} ; K} \geq 0,
$$

which guarantees the derivative (B7) to be between -1 and 0 . Integration gives the inequality

$$
\Delta f_{t}\left(K_{2}\right)-\Delta f_{t}\left(K_{1}\right) \leq K_{1}-K_{2},
$$

where $\Delta f_{t}(K)=\Delta f\left(G_{t}, H_{t} ; K\right)$. We now take $K_{1}=K_{h}(G, H)$ and $K_{2}=K_{h}^{*}(H, G)$ so that in the limit of the sequence, $\lim _{t} \Delta f_{t}\left(K_{1}\right)=0$ and $\lim _{t} \Delta f_{t}\left(K_{2}\right)=R \ln 2$. Equation (35) trivially follows.

\section{APPENDIX C: PROOF OF THEOREM 2}

Theorem 2. Consider a sequence of pairs of weakly dual Ising models defined by pairs of finite binary matrices with mutually orthogonal rows, $G_{t} H_{t}^{T}=0, t \in \mathbb{N}$, where row weights of each $G_{t}$ do not exceed a fixed $m$, CSS distances $d_{H_{t}}$ are increasing with $t$, and the sequence of CSS rates $R_{t} \equiv k_{t} / n_{t}$ converges, $\lim _{t} R_{t}=R$. Then, for any $K \geq 0$ such that $(m-1) \tanh K<1$, the sequence $\Delta f_{t} \equiv\left[\Delta f_{\mathbf{e}}\left(G_{t}, H_{t} ; K\right)\right]_{p}, t \in \mathbb{N}$, converges to $R \ln 2$. 
Proof. The proof is based on the special case of Theorem 1 in the absence of disorder, $p=0$, and the duality relation (33), applied for each pair of matrices, $G_{t}$ and $H_{t}$, with $R_{t}=k_{t} / n_{t}$, and $K$ replaced with its Kramers-Wannier dual, $K^{*}$. The condition on $K$ in Theorem 1 (with $G_{t}$ and $H_{t}$ interchanged) becomes simply $(m-1) \tanh K<1$. Convergence of sequences $\Delta f_{0}\left(H_{t}, G_{t} ; K^{*}\right)$ to 0 and $R_{t}$ to $R$ implies that of the sequence $\Delta f_{0}\left(G_{t}, H_{t} ; K\right)$ to $R \ln 2$.

\section{APPENDIX D: PROOF OF STATEMENT 3}

The proof is based on Theorem 9.1.7 from Ref. 58, which bounds cumulants of a random variable $X$,

$$
\left.\kappa_{r}(X) \equiv \frac{d^{r}}{d t^{r}} \ln \mathbb{E}\left(e^{t X}\right)\right|_{t=0}, r \in\{0,1, \ldots\}
$$

where $X=\sum_{\alpha \in \mathcal{S}} Y_{\alpha}$ is a sum of random variables with a given dependency graph:

Definition 1. A graph $\mathcal{D}$ with vertex set $\mathcal{S}$ is called a dependency graph for the set of random variables $\left\{Y_{\alpha}, \alpha \in \mathcal{S}\right\}$ if for any two disjoint subsets $\mathcal{S}_{1}$ and $\mathcal{S}_{2}$ of $\mathcal{S}$, such that there are no edges in $\mathcal{D}$ connecting an element of $\mathcal{S}_{1}$ and an element of $\mathcal{S}_{2}$, the sets of random variables $\left\{Y_{\alpha}\right\}_{\alpha \in \mathcal{S}_{1}}$ and $\left\{Y_{\alpha}\right\}_{\alpha \in \mathcal{S}_{2}}$ are independent.

The corresponding bound reads as follows:

Lemma 10 (Theorem 9.1.7 from Ref. 58). Let $\left\{Y_{\alpha}\right\}_{\alpha \in \mathcal{S}}$ be a family of random variables with dependency graph $\mathcal{D}$. Denote $N=|\mathcal{S}|$ the number of vertices of $\mathcal{D}$ and $\Delta$ the maximal degree of $\mathcal{D}$. Assume that the variables $Y_{\alpha}$ are uniformly bounded by a constant $A$. Then, for the sum $X=\sum_{\alpha \in \mathcal{S}} Y_{\alpha}$, and for any $s \in\{0,1, \ldots\}$, one has

$$
\left|\kappa_{s}(X)\right| \leq 2^{s-1} s^{s-2} N(\Delta+1)^{s-1} A^{s}
$$

Statement 3. Consider any model in the form (1), with an $(\ell, m)$-sparse $r \times n$ coupling matrix $\Theta$. The coefficients of the HTS expansion of the free energy density satisfy

$$
\left|\kappa_{\mathrm{e}}^{(s)}\left(\Theta ; J, h^{\prime}\right)\right| \leq 2^{s-1} s^{s-2} C(\Delta+1)^{s-1} A^{s},
$$

where $A \equiv \max \left(|J|,\left|h^{\prime}\right|\right)$ and $(\boldsymbol{a})$ with $J$ and $h^{\prime}$ both nonzero, $\Delta=\ell \mathrm{m}$ and $C=r / n+1$, while $(\boldsymbol{b})$ with $h^{\prime}=0, \Delta=(\ell-1) m$ and $C=1$.

Proof of Statement 3. The sth coefficient of the HTS for the free energy $F(\Theta ; K, h)$ is the scaled cumulant $-\kappa_{s}(X) / s !$, where $X=J \sum_{b \in \mathcal{B}} R_{b}$ $+h^{\prime} \sum_{v \in \mathcal{V}} S_{v}$. Define the set of random variables $Y_{\alpha}$ as the union of the set of (scaled) spins $h S_{v}$ and bonds $K R_{b}$, then $\left|Y_{\alpha}\right| \leq A \equiv \max \left(\left|h^{\prime}\right|,|J|\right)$. The corresponding dependency graph $\mathcal{D}$ can be obtained from the bipartite graph defined by the matrix $\Theta$ by connecting any pair of nodes for bonds which share the same spin. In the original bipartite graph, each spin node has up to $\ell$ neighboring bond nodes, and each bond node has up to $m$ neighboring spin nodes. In the modified graph, each bond node also connects with up to $(\ell-1) m$ bond nodes with common spins, which gives the total maximum degree of $\Delta=\ell m$. We also have $N=|\mathcal{V}|+|\mathcal{E}|=r+n$, dividing by $n$ as appropriate for the free energy density, we obtain the bound in part (a). With $h=0$, we can drop the spin nodes from the dependency graph. In this case, the maximum degree is $\Delta^{\prime}=(\ell-1) m$, which gives the result in part (b). Notice that in this case, $N=n$, and the factor $C=(r / n+1)$ is replaced with $C^{\prime}=1$.

\section{APPENDIX E: PROOF OF COROLLARY 4}

Corollary 4. Any infinite sequence of $(\ell, m)$-sparse Ising models, specified in terms of the matrices $\Theta_{j}, j \in \mathbb{N}$, has an infinite subsequence $\Theta_{j(t)}, t \in \mathbb{N}$, where $j: \mathbb{N} \rightarrow \mathbb{N}$ is strictly increasing such that $(\boldsymbol{a})$ for each $s$, the sequence of the coefficients $\kappa_{0}^{(s)}\left(\Theta_{t} ; J, 0\right)$ converges with $t$, and $(\boldsymbol{b})$ the sequence of free energy densities $f\left(\Theta_{j(t)}\right.$; $\left.K\right)$ has a limit, $\varphi_{\Theta}(K)$, which is an analytic function of $K$ in the interior of the circle $|K| \leq\{2 e[(\ell-1) m+1]\}^{-1}$. Here, $e$ is the base of natural logarithm.

Proof. The result in statement $3(\mathrm{~b})$ is uniform in $t$ bound on the coefficients of the HTS,

$$
\begin{aligned}
\frac{\left|\kappa_{s}\left(\Theta_{j}\right)\right|}{s !} & \leq \frac{2^{s-1} s^{s-2}(\Delta+1)^{s-1} J^{s}}{(2 \pi s)^{1 / 2}(s / e)^{s}} \\
& =\frac{1}{\sqrt{8 \pi}(\Delta+1)} \frac{[2 e J(\Delta+1)]^{s}}{s^{5 / 2}}
\end{aligned}
$$

where $\Delta \equiv(\ell-1) m$, and we used the lower bound by Stirling, $r ! \geq(2 \pi r)^{1 / 2}(r / e)^{r}$. The bound (E1) is uniform in the sequence index $j \in \mathbb{N}$. Thus, one can select an infinite subsequence of $\Theta_{j}, \Theta_{j^{\prime}(t)}, t \in \mathbb{N}$, where the function $j^{\prime}: \mathbb{N} \rightarrow \mathbb{N}$ is strictly increasing so that the coefficients 
$\kappa_{m}\left(\Theta_{j^{\prime}(t)}\right)$ for $m=1$ converge with $t$. Selecting an infinite subsequence of the one obtained previously to ensure the convergence of the coefficients $\kappa_{m}$ for $m=2,3, \ldots$, at each step, we obtain an infinite subsequence such that all coefficients $\kappa_{s}$ with $s \leq m$ converge with $t$. The statement in part (a) is obtained in the limit of $m \rightarrow \infty$. The uniform bound (E1) also applies to the cumulants after we take the limit of the obtained subsequence, which implies absolute convergence (and thus analyticity of the limit) of the HTS for free energy density in the circle $|K| \equiv|\beta| J \leq\{2 e[(\ell-1) m+1]\}^{-1}$, which is exactly the statement in part (b).

\section{APPENDIX F: PROOF OF LEMMA 5}

Lemma 5. Consider a sequence of $r_{t} \times n_{t}$ binary matrices $\Theta_{t}$, where $0<r_{t} \leq n_{t}$, and $t \in \mathbb{N}$. For any $M>0$, define a closed interval $I_{M} \equiv[0, M]$. (a) There exists a subsequence $\Theta_{t(i)}, i \in \mathbb{N}$, where the function $t: \mathbb{N} \rightarrow \mathbb{N}$ is strictly increasing, $t(i+1)>t(i)$ for all $i \in \mathbb{N}$, such that the sequence of Ising free energy densities converges for any $K \in I_{M}, f_{i}(K) \equiv f_{0}\left(\Theta_{t(i)} ; K\right) \rightarrow f(K)$. (b) The limit $f(K)$ is a continuous nonincreasing concave function with left and right derivatives uniformly bounded,

$$
-1 \leq f_{+}^{\prime}(K) \leq f_{-}^{\prime}(K) \leq 0
$$

for all $K \in I_{M}$.

Proof. For any $t$, the free energy density $f_{t}(K)=-n_{t}^{-1} \ln Z_{0}\left(G_{t}, K\right)$ is bounded from both sides,

$$
-M \leq r_{t} \ln 2 / n_{t}-K \leq f_{t}(K) \leq r_{t} \ln 2 / n_{t}+K \leq \ln 2+M .
$$

Therefore, we can use a subsequence construction to ensure convergence in any point $K \in I_{M}$. Since the set of rational numbers $\mathbb{Q}$ is countable, we can repeat this construction sequentially on all rational points in $I_{M}$. The resulting infinite sequence $f_{i}(K)$ converges in any rational point $K \in I_{M} \cap \mathbb{Q}$. Furthermore, the derivative of $f_{i}(K)$ is uniformly bounded, $-1 \leq f_{i}^{\prime}(K) \leq 0$. Since the sequence converges on a dense subset of $I_{M}$, this guarantees the existence and the continuity of the limit in the entire interval. Finally, each of $f_{i}(K)$ is concave and nonincreasing; these properties survive the limit although the resulting function may not necessarily be strictly concave. Concavity guarantees the existence of one-sided derivatives. The lower and upper bounds on these derivatives are inherited from those for $f_{i}^{\prime}(K)$.

\section{APPENDIX G: PROOF OF THEOREM 6}

Theorem 6. Let us assume that any one of the following conditions is true:

1. The transition at $T_{c}^{\prime}(G)$ is discontinuous or has a divergent specific heat;

2. The derivative of $\Delta f(K)=f_{G}(K)-f_{H^{*}}(K)$ is discontinuous at $K_{h} \equiv K_{h}(G, H)$, or the derivative of $\Delta f(K)$ is continuous at $K_{h}$, but its second derivative diverges at $K_{h}$;

3. Summation over homological defects does not increase the critical temperature, $T_{c}\left(G^{*}\right) \leq T_{c}(H)$.

Then, the Kramers-Wannier dual of the critical temperatures $T_{c}(H)$ satisfies

$$
T_{c}^{*}(H) \leq T_{h}(G, H) .
$$

Proof. There are three mutually exclusive possibilities: (a) $T_{c}^{\prime}(G)<T_{h}(G, H)$, (b) $T_{c}^{\prime}(G)>T_{h}(G, H)$, and (c) $T_{c}^{\prime}(G)=T_{h}(G, H)$. In the case (a), $T_{c}^{*}(H)=T_{c}^{\prime}(G)$ since the functions $f_{G}(K)$ and $f_{H^{*}}(K)$ coincide in the homological region, i.e., for $K>K_{h}(G, H)$; Eq. (41) is satisfied. In the case (b), $T_{c}^{*}(H)=T_{h}(G, H)$, in order to recover the nonanalyticity point for the homological difference; Eq. (41) is saturated. The goal of the conditions is to deal with the case (c) which implies $T_{c}^{*}(H) \geq T_{h}(G, H)$; a strict inequality would violate Eq. (41). In the following, we assume (c).

Condition 1 implies that the (negative) curvature of $f_{G}(K)$ must diverge at $K_{h}=K_{c}^{\prime}(G)$, which must be compensated by a divergent curvature of $f_{H^{*}}(K)$ in order to make $\Delta f(K)$ strictly convex in this point. In this case, $T_{c}^{*}(H)=T_{c}^{\prime}(G)$; Eq. (41) is saturated.

Condition 2 does the same since the divergent positive curvature of $\Delta f(K)$ at $K_{h}$ can only come from $f_{H^{*}}(K)$.

Condition 3 is equivalent by duality to $T_{c}^{\prime}(G) \geq T_{c}^{*}(H)$, which again gives Eq. (41), since we assumed (c).

\section{APPENDIX H: PROOF OF THE LOWER BOUND FOR TENSION}

On an infinite locally planar transitive graph $\mathcal{G}$, we would like to prove the following bound for the asymptotic defect tension (43),

$$
\frac{d \tau(K)}{d K} \geq 2[m(K)]^{2}
$$

the same inequality as has been previously proved on $\mathbb{Z}^{D}$ in Ref. 4 . This inequality is a trivial consequence of the following lemma, which gives a version of Eq. (7) from Ref. 4 suitable to constructing a bound for the defect tension defined by Eq. (15). 
Lemma 11. Let $\mathcal{G}=(\mathcal{V}, \mathcal{E})$ be a finite transitive graph, and $G$ be the corresponding vertex-edge incidence matrix with $n=|\mathcal{E}|$ columns. Take a binary vector $\mathbf{e} \in \mathbb{F}_{2}^{n}$ selecting a set of edges $\mathcal{E}_{\mathbf{e}} \subset \mathcal{E}$ of size $\left|\mathcal{E}_{\mathbf{e}}\right|=\operatorname{wgt}(\mathbf{e})$, and a set of vertices $\mathcal{A} \subset \mathcal{V}$ of twice the size, $|\mathcal{A}|=2 \mathrm{wgt}(\mathbf{e})$, such that the graph contains edge-disjoint paths connecting each edge to exactly two vertices in $\mathcal{A}$. Then, for the Ising model defined on the same graph, at any $K, h \geq 0$, the free energy increment $\delta_{\mathbf{e}}(K) \equiv F_{\mathbf{e}}(G ; K, h)-F_{\mathbf{0}}(G ; K, h)$ associated with the defect $\mathbf{e}$ satisfies

$$
\frac{d \delta_{\mathbf{e}}(K)}{d K} \geq\left\langle S_{i}\right\rangle_{0} \sum_{v \in \mathcal{A}}\left\langle S_{v}\right\rangle_{\mathbf{e}}
$$

where the average $\left\langle S_{v}\right\rangle_{\mathbf{e}}$ is calculated in the presence of the defect $\boldsymbol{e}$; by transitivity, $\left\langle S_{i}\right\rangle_{\mathbf{0}}$ is independent of $i \in \mathcal{V}$.

Proof. The proof is based on two inequalities,

$$
\left\langle S_{\mathcal{A}} S_{\mathcal{B}}\right\rangle_{\mathbf{0}} \pm\left\langle S_{\mathcal{A}} S_{\mathcal{B}}\right\rangle_{\mathbf{e}} \geq\left|\left\langle S_{\mathcal{A}}\right\rangle_{0}\left\langle S_{\mathcal{B}}\right\rangle_{\mathbf{e}} \pm\left\langle S_{\mathcal{A}}\right\rangle_{\mathbf{e}}\left\langle S_{\mathcal{B}}\right\rangle_{0}\right|
$$

where $\mathcal{A} \subset \mathcal{V}$ and $\mathcal{B} \subset \mathcal{V}$ are sets of vertices. The inequality with the lower (negative) signs is the Lebowitz comparison inequality, ${ }^{2}$ while the inequality with the upper signs can be proved using the same technique. In the case of an Ising model on a graph $\mathcal{G}=(\mathcal{V}, \mathcal{E})$, we have

$$
\frac{d \delta_{\mathbf{e}}(K)}{d K}=\sum_{i j=b \in \mathcal{E}}\left[\left\langle S_{i} S_{j}\right\rangle_{\mathbf{0}}-(-1)^{e_{b}}\left\langle S_{i} S_{j}\right\rangle_{\mathbf{e}}\right] .
$$

Applying Eq. (H3) for each term separately, with the help of transitivity, $\left\langle S_{i}\right\rangle_{0} \equiv m_{0} \geq 0, i \in \mathcal{V}$, one gets

$$
\frac{d \delta_{\mathbf{e}}(K)}{d K} \geq m_{0} \sum_{b=i j \in \mathcal{E}}\left|m_{i}^{\prime}-(-1)^{e_{b}} m_{j}^{\prime}\right|,
$$

where $m_{i}^{\prime} \equiv\left\langle S_{i}\right\rangle_{\mathbf{e}}$. The statement of the lemma is obtained by noticing that for a path connecting 1 and $f$,

$$
\left|m_{1}^{\prime}-m_{2}^{\prime}\right|+\left|m_{2}^{\prime}-m_{3}^{\prime}\right|+\cdots+\left|m_{f-1}^{\prime}-m_{f}^{\prime}\right| \geq m_{f}^{\prime}-m_{1}^{\prime}
$$

which allows us to trade wgt(e) terms with + signs in the rhs of Eq. (H4) for the sum of magnetizations $m_{v}^{\prime}$ on the 2 wgt(e) vertices from $\mathcal{A}$.

\section{REFERENCES}

${ }^{1}$ R. Griffiths, "Rigorous results and theorems," in Phase Transitions and Critical Phenomena, Exact results Vol. 1, edited by C. Domb and M. S. Green (Academic Press, London, 1972), Chap. 2, pp. 7-109.

2J. L. Lebowitz, “Coexistence of phases in Ising ferromagnets," J. Stat. Phys. 16, 463-476 (1977).

${ }^{3}$ C. Gruber and J. L. Lebowitz, "On the equivalence of different order parameters and coexistence of phases for Ising ferromagnet. II," Commun. Math. Phys. 59, $97-108$ (1978).

${ }^{4} J$. L. Lebowitz and C. E. Pfister, “Surface tension and phase coexistence," Phys. Rev. Lett. 46, 1031-1033 (1981).

${ }^{\mathbf{5}}$ R. Lyons, “The Ising model and percolation on trees and tree-like graphs,” Commun. Math. Phys. 125, $337-353$ (1989).

${ }^{6}$ R. Lyons, "Phase transitions on nonamenable graphs," J. Math. Phys. 41, 1099-1126 (2000); e-print arXiv:math/9908177.

${ }^{7}$ P. Gerl, "Random walks on graphs with a strong isoperimetric property," J. Theor. Probab. 1, 171-187 (1988).

${ }^{8}$ R. M. Burton and M. Keane, "Density and uniqueness in percolation,” Commun. Math. Phys. 121, 501-505 (1989).

${ }^{9}$ I. Benjamini and O. Schramm, "Percolation beyond $Z^{d}$, many questions and a few answers," Electron. Commun. Probab. 1, 71-82 (1996).

${ }^{10} \mathrm{O}$. Häggström and J. Jonasson, “Uniqueness and non-uniqueness in percolation theory," Probab. Surv. 3, 289-344 (2006).

${ }^{11}$ I. Benjamini and O. Schramm, "Percolation in the hyperbolic plane," J. Am. Math. Soc. 14, 487-507 (2001).

${ }^{12}$ J. Jonasson and J. E. Steif, “Amenability and phase transition in the Ising model,” J. Theor. Probab. 12, 549-559 (1999).

${ }^{13}$ C. M. Fortuin and P. W. Kasteleyn, “On the random-cluster model: I. Introduction and relation to other models," Physica 57, 536-564 (1972).

${ }^{14}$ C. M. Fortuin, “On the random-cluster model: II. The percolation model," Physica 58, 393-418 (1972).

${ }^{15}$ H. R. Schonmann, "Multiplicity of phase transitions and mean-field criticality on highly non-amenable graphs," Commun. Math. Phys. 219, 271-322 (2001).

${ }^{16}$ O. Häggström, J. Jonasson, and R. Lyons, “Explicit isoperimetric constants and phase transitions in the random-cluster model," Ann. Probab. 30, 443-473 (2002).

${ }^{17}$ N. B. Birrel and P. C. W. Davies, Quantum Fields in Curved Space (Cambridge University Press, Cambridge, UK, 1982).

${ }^{18}$ G. Cognola, K. Kirsten, and S. Zerbini, “One-loop effective potential on hyperbolic manifolds,” Phys. Rev. D 48, 790-799 (1993).

${ }^{19}$ R. Camporesi, “ $\zeta$-function regularization of one-loop effective potentials in anti-de Sitter spacetime,” Phys. Rev. D 43, 3958-3965 (1991).

${ }^{20}$ G. Miele and P. Vitale, “Three-dimensional Gross-Neveu model on curved spaces," Nucl. Phys. B 494, 365-387 (1997).

${ }^{21}$ B. Doyon, "Two-point correlation functions of scaling fields in the Dirac theory on the Poincaré disk," Nucl. Phys. B 675, 607-630 (2003). 
${ }^{22}$ G. Evenbly and G. Vidal, “Tensor network states and geometry,” J. Stat. Phys. 145, 891-918 (2011).

${ }^{23}$ H. Matsueda, M. Ishihara, and Y. Hashizume, “Tensor network and a black hole," Phys. Rev. D 87, 066002 (2013).

${ }^{24}$ D. R. Nelson, “Order, frustration, and defects in liquids and glasses," Phys. Rev. B 28, 5515-5535 (1983).

${ }^{25}$ G. Tarjus, S. A. Kivelson, Z. Nussinov, and P. Viot, "The frustration-based approach of supercooled liquids and the glass transition: A review and critical assessment," J. Phys.: Condens. Matter 17, R1143 (2005).

${ }^{26}$ V. Vitelli, J. B. Lucks, and D. R. Nelson, “Crystallography on curved surfaces,” Proc. Natl Acad. Sci. U. S. A. 103, 12323-12328 (2006).

${ }^{27}$ F. Sausset and G. Tarjus, "Periodic boundary conditions on the pseudosphere," J. Phys. A: Math. Theor. 40, 12873 (2007).

${ }^{28}$ L. Giomi and M. Bowick, "Crystalline order on Riemannian manifolds with variable Gaussian curvature and boundary," Phys. Rev. B 76, 054106 (2007).

${ }^{29}$ A. M. Turner, V. Vitelli, and D. R. Nelson, "Vortices on curved surfaces," Rev. Mod. Phys. 82, 1301-1348 (2010).

${ }^{30}$ N. A. Garcia, A. D. Pezzutti, R. A. Register, D. A. Vega, and L. R. Gomez, "Defect formation and coarsening in hexagonal 2D curved crystals," Soft Matter 11, 898-907 (2015).

${ }^{31}$ D. Benedetti, "Critical behavior in spherical and hyperbolic spaces," J. Stat. Mech.: Theor. Exp. 2015, P01002.

${ }^{32}$ R. Cohen, K. Erez, D. ben-Avraham, and S. Havlin, "Resilience of the internet to random breakdowns," Phys. Rev. Lett. 85, 4626-4628 (2000).

${ }^{33}$ R. Albert and A.-L. Barabási, "Statistical mechanics of complex networks," Rev. Mod. Phys. 74, 47-97 (2002).

${ }^{34}$ P. Gai and S. Kapadia, "Contagion in financial networks," Proc. R. Soc. A 466, 2401-2423 (2010).

${ }^{35}$ K. Börner, S. Sanyal, and A. Vespignani, “Network science,” Annu. Rev. Inf. Sci. Technol. 41, 537-607 (2007).

${ }^{36}$ L. M. Sander, C. P. Warren, I. M. Sokolov, C. Simon, and J. Koopman, "Percolation on heterogeneous networks as a model for epidemics," Math. Biosci. 180, 293-305 (2002).

${ }^{37}$ L. da Fontoura Costa, O. N. Oliveira, G. Travieso, F. A. Rodrigues, P. R. Villas Boas, L. Antiqueira, M. P. Viana, and L. E. Correa Rocha, “Analyzing and modeling real-world phenomena with complex networks: A survey of applications," Adv. Phys. 60, 329-412 (2011).

${ }^{38}$ L. Danon, A. P. Ford, T. House, C. P. Jewell, M. J. Keeling, G. O. Roberts, J. V. Ross, and M. C. Vernon, "Networks and the epidemiology of infectious disease," Interdiscip. Perspect. Infect. Dis. 2011, 284909.

${ }^{39}$ E. Dennis, A. Kitaev, A. Landahl, and J. Preskill, “Topological quantum memory,” J. Math. Phys. 43, 4452 (2002).

${ }^{40}$ A. A. Kovalev and L. P. Pryadko, "Spin glass reflection of the decoding transition for quantum error-correcting codes," Quantum Inf. Comput. 15, 0825 (2015); e-print arXiv:1311.7688.

${ }^{41}$ A. A. Kovalev, S. Prabhakar, I. Dumer, and L. P. Pryadko, "Numerical and analytical bounds on threshold error rates for hypergraph-product codes," Phys. Rev. A 97, 062320 (2018); e-print arXiv:1804.01950.

${ }^{42}$ C. C. Wu, "Ising models on hyperbolic graphs II," J. Stat. Phys. 100, 893-904 (2000).

${ }^{43}$ F. J. Wegner, "Duality in generalized Ising models and phase transitions without local order parameters," J. Math. Phys. 12, 2259 (1971).

${ }^{44} \mathrm{R}$. Tanner, “A recursive approach to low complexity codes," IEEE Trans. Inf. Theory 27, 533-547 (1981).

${ }^{45}$ J. K. Percus, “Correlation inequalities for Ising spin lattices,” Commun. Math. Phys. 40, 283-308 (1975).

${ }^{46}$ S. B. Shlosman, "Correlation inequalities and their applications," J. Sov. Math. 15, 79-101 (1981).

${ }^{47}$ R. B. Griffiths, “Correlations in Ising ferromagnets. I,” J. Math. Phys. 8, 478 (1967).

${ }^{48}$ D. G. Kelly and S. Sherman, "General Griffiths' inequalities on correlations in Ising ferromagnets," J. Math. Phys. 9, 466-484 (1968).

${ }^{49}$ H. A. Kramers and G. H. Wannier, "Statistics of two-dimensional ferromagnet. Part I," Phys. Rev. 60, 252-262 (1941).

${ }^{50} \mathrm{H}$. Nishimori, Statistical Physics of Spin Glasses and Information Processing: An Introduction (Clarendon Press, Oxford, 2001).

${ }^{51}$ D. Gottesman, "Stabilizer codes and quantum error correction," Ph.D. thesis, Caltech, 1997.

${ }^{52}$ A. R. Calderbank, E. M. Rains, P. M. Shor, and N. J. A. Sloane, “Quantum error correction via codes over GF(4)," IEEE Trans. Inf. Theory 44, 1369-1387 (1998).

${ }^{53}$ M. A. Nielsen and I. L. Chuang, Quantum Computation and Quantum Infomation (Cambridge University Press, Cambridge, MA, 2000).

${ }^{54}$ J. Preskill, Course Information for Physics 219/Computer Science 219 Quantum Computation (Caltech, 2000).

${ }^{55}$ A. R. Calderbank and P. W. Shor, "Good quantum error-correcting codes exist," Phys. Rev. A 54, 1098-1105 (1996).

${ }^{56}$ A. M. Steane, "Simple quantum error-correcting codes," Phys. Rev. A 54, 4741-4751 (1996).

${ }^{57}$ Notice that any other construction of the dual matrix would at most change the partition function multiplicatively by a power of two.

${ }^{58}$ V. Féray, P.-L. Méliot, and A. Nikeghbali, Mod- $\phi$ Convergence I: Normality Zones and Precise Deviations (Springer International Publishing, Cham, 2016); e-print arXiv:1304.2934v4.

${ }^{59}$ I. Benjamini and O. Schramm, "Recurrence of distributional limits of finite planar graphs," Electron. J. Probab. 6, 23-1-23-13 (2001); e-print arXiv:math/0011019.

${ }^{60}$ C. Borgs, J. Chayes, J. Kahn, and L. Lovász, "Left and right convergence of graphs with bounded degree," Random Struct. Algorithms 42, 1-28 (2013); e-print arXiv:1002.0115.

${ }^{61}$ L. M. Lovász, “A short proof of the equivalence of left and right convergence for sparse graphs," Eur. J. Combinatorics 53, 1-7 (2016).

${ }^{62}$ Phase Transitions and Critical Phenomena, edited by C. Domb and M. S. Green (Academic, London, 1974), Vol. 3.

${ }^{63}$ S. Bravyi and B. Terhal, "A no-go theorem for a two-dimensional self-correcting quantum memory based on stabilizer codes," New J. Phys. 11, 043029 (2009).

${ }^{64}$ S. Bravyi, D. Poulin, and B. Terhal, "Tradeoffs for reliable quantum information storage in 2D systems," Phys. Rev. Lett. 104, 050503 (2010); e-print arXiv:0909.5200.

${ }^{65} \mathrm{~J}$.-P. Tillich and G. Zemor, "Quantum LDPC codes with positive rate and minimum distance proportional to $\sqrt{n}$," in Proceedings IEEE International Symposium on Information Theory (ISIT) (IEEE, 2009), pp. 799-803.

${ }_{66}^{6}$ A. A. Kovalev and L. P. Pryadko, “Quantum Kronecker sum-product low-density parity-check codes with finite rate," Phys. Rev. A 88, 012311 (2013).

${ }^{67}$ W. Zeng and L. P. Pryadko, "Higher-dimensional quantum hypergraph-product codes," Phys. Rev. Lett. 122, 230501 (2018); e-print arXiv:1810.01519.

${ }^{68}$ L. Guth and A. Lubotzky, "Quantum error correcting codes and 4-dimensional arithmetic hyperbolic manifolds," J. Math. Phys. 55, 082202 (2014); e-print arXiv:1310.5555.

${ }^{69}$ A. Montanari, E. Mossel, and A. Sly, “The weak limit of Ising models on locally tree-like graphs,” Probab. Theory Relat. Fields 152, 31-51 (2012).

${ }^{70} \mathrm{~J}$. Širáň, “Triangle group representations and constructions of regular maps," Proc. London Math. Soc. 82, 513-532 (2001).

${ }^{71}$ N. Delfosse and G. Zémor, "Quantum erasure-correcting codes and percolation on regular tilings of the hyperbolic plane," in Information Theory Workshop (ITW), 2010 (IEEE, 2010), pp. 1-5. 
${ }^{72}$ N. Delfosse and G. Zémor, "Upper bounds on the rate of low density stabilizer codes for the quantum erasure channel," Quantum Info. Comput. 13, 793-826 (2013); e-print arXiv:1205.7036.

${ }^{73} \mathrm{~N}$. Delfosse, "Tradeoffs for reliable quantum information storage in surface codes and color codes," in IEEE International Symposium on Information Theory Proceedings (ISIT), 2013 (IEEE, 2013), pp. 917-921.

${ }^{74}$ N. Delfosse and G. Zémor, “A homological upper bound on critical probabilities for hyperbolic percolation,” Ann. Inst. Henri Poincaré D 3, 139-161 (2016); e-print arXiv:1408.4031.

${ }^{75}$ N. P. Breuckmann and B. M. Terhal, “Constructions and noise threshold of hyperbolic surface codes,” IEEE Trans. Inf. Theory 62, 3731-3744 (2016); e-print arXiv: 1506.04029 .

${ }^{76}$ N. P. Breuckmann, C. Vuillot, E. Campbell, A. Krishna, and B. M. Terhal, “Hyperbolic and semi-hyperbolic surface codes for quantum storage,” Quantum Sci. Technol. 2, 035007 (2017).

${ }^{77}$ N. P. Breuckmann, "Homological quantum codes beyond the toric code," Ph.D. thesis, RWTH Aachen University, 2017; e-print arXiv:1802.01520.

${ }^{78}$ A. Y. Kitaev, "Fault-tolerant quantum computation by anyons," Ann. Phys. 303, 2 (2003).

${ }^{79}$ N. Metropolis, A. W. Rosenbluth, M. N. Rosenbluth, A. H. Teller, and E. Teller, "Equation of state calculations by fast computing machines," J. Chem. Phys. 21, 1087-1092 (1953).

${ }^{80}$ U. Wolff, "Collective Monte Carlo updating for spin systems," Phys. Rev. Lett. 62, 361-364 (1989).

${ }^{81}$ K. Binder, "Finite size scaling analysis of Ising model block distribution functions," Z. Phys. B: Condens. Matter 43, 119-140 (1981).

${ }^{82}$ GAP, GAP-Groups, Algorithms, and Programming, Version 4.8.10, The GAP Group, 2018.

${ }^{83}$ I. Dumer, A. A. Kovalev, and L. P. Pryadko, "Numerical techniques for finding the distances of quantum codes," in IEEE International Symposium on Information Theory Proceedings (ISIT), 2014 (IEEE, Honolulu, HI, 2014), pp. 1086-1090.

${ }^{84}$ I. Dumer, A. A. Kovalev, and L. P. Pryadko, "Distance verification for classical and quantum LDPC codes," IEEE Trans. Inf. Theory 63, 4675-4686 (2017).

${ }^{85}$ I. Dumer, A. A. Kovalev, and L. P. Pryadko, "Thresholds for correcting errors, erasures, and faulty syndrome measurements in degenerate quantum codes," Phys. Rev. Lett. 115, 050502 (2015); e-print arXiv:1412.6172.

${ }^{86}$ M. E. Fisher, “Critical temperatures of anisotropic Ising lattices. II. General upper bounds," Phys. Rev. 162, 480-485 (1967).

${ }^{87}$ J. L. Lebowitz, "Bounds on the correlations and analyticity properties of ferromagnetic Ising spin systems," Commun. Math. Phys. 28, 313-321 (1972).

${ }^{88}$ J. Bricmont, J. L. Lebowitz, and C. E. Pfister, “On the surface tension of lattice systems," Ann. N. Y. Acad. Sci. 337, 214-223 (1980).

${ }^{89}$ F. J. MacWilliams and N. J. A. Sloane, The Theory of Error-Correcting Codes (North-Holland, Amsterdam, 1981). 